\title{
A thermodynamics viscoelastic constitutive model for shape memory
}

\section{polymers}

Jianming Guo ${ }^{\mathrm{a}, \mathrm{b}, \mathrm{c}}$,Jingbiao Liu ${ }^{\mathrm{a}}$, Zhenqing Wang ${ }^{\mathrm{a},{ }^{*}}, \mathrm{Xiaofu} \mathrm{He}^{\mathrm{b}}$, Lifeng $\mathrm{Hu}^{\mathrm{b}}$, and Liyong Tong ${ }^{\mathrm{c}}$

${ }^{a}$ College of Aerospace and Civil Engineering, Harbin Engineering University, Harbin, China

${ }^{\mathrm{b}}$ Jiancheng group co., ltd, Harbin, China

${ }^{c}$ School of Aerospace, Mechanical and Mechatronic Engineering, The University of Sydney, NSW 2006 Australia

* Corresponding author: Tel: +8613704519999 E-mail: wangzhenqing@ hrbeu.edu.cn

\begin{abstract}
In this study, a thermodynamics viscoelastic constitutive model was developed to describe behaviors of the typical thermo-mechanical cycle experimentations. In this model, the changes of thermodynamic and mechanics for SMPs in cooling/heating process were described clearly and the irreversible deformations were calculated. A new type of microstructure of SMPs was proposed and new definitions of phases: active phase and frozen phase were set forth in the present study. The new thermodynamics viscoelastic model is series by two parts: mechanical parts and thermal parts. A new transform equation for the transformation of frozen phase was proposed, and the transform delay time and cooling/heating rate were considered in this transform equation. A transform equation based on the transformation of frozen phase was proposed to describe the change of thermal expansion coefficient of SMPs. Finally, the typical thermo-mechanical cycle experimentation processes were calculated using the new thermodynamics viscoelastic constitutive model. Through the comparison between the calculated results and experimental results, the rationality and accuracy of the new thermodynamics viscoelastic constitutive model was finally verified.
\end{abstract}

Keywords: constitutive model; shape memory polymers; thermodynamics; viscoelastic

\section{Introduction}

Shape memory polymers (SMPs) is one type of new shape memory materials [1]. It was first developed in nineteen-eighties [2], and thereafter various kinds of SMPs have been developed and a vast majority of research works have been conducted [3-5]. As one important type of stimuli-responsive materials, SMPs recovers its original shape from a temporary one under an appropriate stimulation, such as, temperature, $\mathrm{PH}$, electric, light, enzyme or magnetic fields [6-8]. In compared to shape memory ceramics (SMCs) [9] and shape memory alloys 
(SMAs) [10], SMPs has significant advantages, such as biocompatibility, light weight, high dimensional deformability, superior process ability and low cost [11-14]. In recent years, some successful applications, such as, shape memory hybrids [15], crawling robots [16], selfassembly robots [17] and self-folding machines [18], have been reported in academia and industries.

In order to effectively describe the shape memory effect (SME) and to enable its wide applications, it is necessary to establish appropriate constitutive models for SMPs. In 1997, Tobushi et al developed a linear viscoelastic constitutive model to describe the thermodynamics behaviors of SMPs by introducing an irrecoverable slip element [19]. Further to this research, Tobushi et al developed a one-dimensional nonlinear constitutive model [20]. Liu et al divided the behavior of SMPs into two parts: the active phase at high temperature and the frozen phase at low temperature, and develop a three-dimensional linear constitutive model [21]. Based on this research, Chen and Lagoudas in 2008 [22, 23] developed a constitutive theory for SMPs under large deformations and a linear constitutive theory for SMPs under small deformations. Behl and Lendlein $[3,24]$ indicated that the molecular interaction is the main reason for SME of SMPs. Through comparison between the physical nature of crystallization and the melting of polymers, Wang et al proposed a three-dimensional constitutive model that corresponds to Liu's experimental results [25]. During this time period, Zhou et al developed a glass transition model for SMPs and a three-dimensional macromechanical constitutive model for SMPs [26, 27]. In 2012, Baghani et al presented a threedimensional constitutive model for SMPs subject to time-dependent multiaxial thermodynamics loadings under small deformations [28]. Recently Guo et al indicated that stress is an important influence factor on phase transition for SMPs in cooling and heating process, and a three-dimensional constitutive model and developed a phase transition model for semi-crystallize SMPs [29, 30].

In the studies of SMPs, viscoelastic constitutive model is an important component of constitutive models of SMPs. Generally speaking these viscoelastic constitutive models adopt a variable or an element in three- or four-element viscoelastic models [19, 31-35]. These viscoelastic constitutive models describe the thermodynamics behaviors of SMPs effectively. However the microscopic mechanism of the SME in SMPs couldn't be explained in these models. Since the theory of phase transformation between active and frozen phases in SMPs was proposed by Liu et al [21], many different viscoelastic constitutive models were developed to elaborate the SME of SMPs. These viscoelastic constitutive models adopt series structure models to represent the active and frozen phases [29, 30, 36]. This is due to the assumed 
condition in the phase transformation theory [21] is that the heating/cooling rate is so slowly that the active phase and the frozen phase appear in SMPs evenly. However, in practical experiments, the temperature of SMPs changes from its surface to inner in the heating/cooling process, and the corresponding phase transforms from surface to inner of SMPs. Therefore, a viscoelastic constitutive with parallel structures, which represents active phase and frozen phase respectively, maybe deemed as a new method to describe the SME of SMPs.

In the present study, the microstructure of a semi-crystalline SMP [37] was considered and a new composite system of SMPs was proposed. In this composite system, the SMPs are assumed to be composed of two phases: one is the active phase with a constant network, and the other one is the frozen phase which transforms between free and frozen states. Additionally, a viscoelastic constitutive model with parallel structures representing two phases was developed. According to the thermodynamics theory of polymers [38-40], the developed thermodynamics viscoelastic constitutive model is in series by two parts: the mechanical parts and the thermal parts. The mechanical parts are in parallel and represented by a four-element structure and a three-element structure, which represent the active phase and frozen phase, respectively. The thermal parts consist of two parallel one-element structures representing the thermal expansion of SMPs in high temperature and low temperature respectively. Finally, for the sake of verifying the accuracy of the present new viscoelastic constitutive model, we conducted a comparison between the constitutive models and experimental results of Tobushi et al $[19,20,41]$ and Liu et al [21] and the present model.

\section{Basics of a new thermodynamics viscoelastic constitutive model}

SMPs possess shape memory properties demonstrated by experiments of various thermo-mechanical loading cycles [21, 41], which include the following typical steps: 1) SMPs are deformed by an external force at high temperature; 2) the deformation of SMPs are held unchanged when temperature is lowered; 3) the external force is then removed; 4) the original configuration of SMPs is recovered freely when reheated. Shape memory properties of SMPs are believed to be due to their molecular structures comprising of amorphous segments each of which owns an appropriate cross-linking density. When SMPs are exposed to temperature higher than transition temperature, the amorphous segments are flexible; and SMPs turn into rubber state when exposed to temperature lower than its transition temperature [25, 42]. To describe this microscopic mechanism of the SME in SMPs, SMPs are typically modeled with a composite consisting of two phases, an active phase and an inactive (frozen) phase. In [43], 
similar to the microstructure of unidirectional fiber reinforced polymeric composite, the active phase is represented by individual fibers and the inactive phase by the polymeric resin. In [21], a particle reinforced composite microstructure was adopted, in which particles represent the frozen phase and polymeric resin denotes the active phase. Both models have a common characteristic that the two phases are evenly distributed in SMPs, and all the transitions between the active and frozen phases start/end at the same time. However, in experiment, the temperature of SMPs changes from its surface to interior in heating/cooling process, and the corresponding phase transitions also occurs from surface to interior.

Based on the microstructure of semi-crystalline SMPs [37] and the temperature transformation of SMPs in experiments, the microstructure of SMPs is assumed in this study to take the form of a composite system of active and frozen phases as shown in Fig 1. In this composite system, it is assumed that the active phase be composed of networks just like the networks in cross-linked polymer and the frozen phase be a material which transforms between the free and frozen states as in crystalline polymer. When temperature is changed from high to low, the frozen phase transforms from its free state to frozen state. In this process, the mechanical state of the active phase is considered as invariable. This is because the networks are believed to be so strong that the state transformation of the frozen phase does not affect the networks, and the internal stress of the networks remains constant. The mechanical state of the active phase is considered as invariable in heating process. In addition, the transition of the frozen phase on the surface and in the interior of a SMP are assumed to be inconsistent or different at a given time, and happens gradually from surface to interior of a SMP in cooling/heating process. Furthermore, the thermal expansion of a polymer is believed to be a non-ignorable factor in the thermal-mechanical behavior of SMPs in cooling/heating process and will be considered in the present study.

According to the thermal theory of polymers [38-40] and the microstructure of SMPs in Fig 1, the structural diagram of the present new thermodynamics constitutive model is shown in Fig 2. The present model is assumed to be composed of the mechanical and thermal parts arranged in a series form. The mechanical part consists of the active and frozen phases arranged in parallel. In this part, the active phase is constant or fixed whereas the frozen phase transforms between free and frozen states. Compared to the active phase with network structures, the mechanical properties of the frozen phase in free state are small; thus the mechanical properties of active phase are assumed to be the mechanical properties of SMPs at high temperature. The thermal part has two components also arranged in parallel characterizing the thermal expansion of SMPs in high and low temperature respectively. In 
this part, the coefficient of thermal expansion of SMPs in high or low temperature is assumed to be the coefficient of thermal expansion of the composite system composed of both active and frozen phases in free or frozen state, respectively. The thermal expansion of SMPs is dependent on the transform of the frozen phase between free and frozen states.

Based on the viscoelasticity theory of polymer [44-46] and the characteristics of the two phases, we propose a new thermodynamics viscoelastic constitutive model as schematically shown in Fig 3. In the present model, the active and frozen phases are represented by a four-element system and a three-element system respectively, and the two one-element systems represent thermal expansion of SMPs in high and low temperatures.

The four-element system for the active phase can be described mathematically by the following constitutive equation:

$$
\sigma+p_{1} \dot{\sigma}+p_{2} \ddot{\sigma}=q_{1} \dot{\varepsilon}+q_{2} \ddot{\varepsilon}
$$

where $p_{1}=\frac{E_{1 a}\left(\mu_{1 a}+\mu_{2 a}\right)+E_{2 a} \mu_{1 a}}{E_{1 a} E_{2 a}}, p_{2}=\frac{\mu_{1 a} \mu_{2 a}}{E_{1 a} E_{2 a}}, q_{1}=\mu_{2 a}$ and $q_{2}=\frac{\mu_{1 a} \mu_{2 a}\left(E_{1 a}+E_{2 a}\right)}{E_{1 a} E_{2 a}}$, and $\sigma$ is the stress of the active phase, $\varepsilon$ is the strain of the active phase, $E_{1 a}$ and $E_{2 a}$ are the Modulus of two spring elements in the four-element structure, $\mu_{1 a}$ and $\mu_{2 a}$ are the viscosity of two dashpot elements in the four-element structure.

For the three-element system of the frozen phase, the mathematical equation is given by:

$$
\sigma+P_{1} \dot{\sigma}=Q_{0} \varepsilon+Q_{1} \dot{\varepsilon}
$$

where $P_{1}=\frac{\mu_{f}}{E_{1 f}}, Q_{0}=E_{2 f}$ and $Q_{1}=\frac{\mu_{f}\left(E_{1 f}+E_{2 f}\right)}{E_{1 f}}, \sigma$ is the stress of the frozen phase in frozen state, $\varepsilon$ is the strain of the frozen phase in frozen state, $E_{1 f}$ and $E_{2 f}$ are the Modulus of two spring elements in the three-element structure, $\mu_{f}$ is the viscosity of dashpot element in the three-element structure.

For the two one-element systems for thermal expansion of SMPs in high and low temperatures, the thermal expansion can be expressed as:

$$
\varepsilon_{T_{h}}=\alpha_{T_{h}} \Delta T, \quad \varepsilon_{T_{l}}=\alpha_{T_{l}} \Delta T
$$

where $\varepsilon_{T_{h}}$ and $\varepsilon_{T_{l}}$ are the thermal expansion strains of the SMPs in high and low temperatures respectively, $\alpha_{T_{h}}$ and $\alpha_{T_{l}}$ are the thermal expansion coefficients of the SMPs in high and low temperature, and $\Delta T$ is the temperature difference. 
As indicated by Wang et al [25], the frozen transition process of SMPs is analogous to the process of crystallization in the semi-crystallization polymers, therefore, the crystallization theory $[47,48]$ was adopted in their model; and hence the transformation of frozen phase can be expressed as follows

$$
1-\phi_{f}=\phi \exp \left(-\left(k T_{\text {tran }} /(T-\tau \beta)\right)^{m} / \beta^{n}\right)
$$

where $\phi_{f}$ is the transformation ratio of the frozen phase, $\phi$ is the final transformation ratio, $k$ is the similarity coefficient between the transition process of SMPs and the process of crystallization, $T_{\text {tran }}$ is the transition temperature of polymer, $T$ is the testing temperature, $\tau$ is time delay which is dependent on the microstructure of the frozen phase and the cooling/heating rate, $\beta$ is the cooling/heating rate, and $m$ and $n$ are the Ozawa exponent and Avrami exponent, respectively.

In the thermal part of the present model, the thermal expansions of SMPs in high and low temperatures are assumed to be in parallel in this composite system and they depend on the transformation of the frozen phase between the free states and frozen states. The coefficient of thermal expansion of the system can be given by:

$$
\alpha=\left(1-\phi_{f}\right) \alpha_{T_{h}}+\phi_{f} \alpha_{T_{l}}
$$

where $\alpha$ is the coefficient of thermal expansion of the composite system, $\phi_{f}$ is the transformation ratio of the frozen phase, $\alpha_{T_{h}}$ and $\alpha_{T_{l}}$ are the coefficients of thermal expansion of the composite system at high and low temperature respectively.

\section{Detailed model formulations for two cases}

To explain in details how to use the present new basic viscoelastic constitutive model for SMPs, we present detailed model formulations for the two cases of the thermo-mechanical loading cycles reported in the experiments in $[19,20,41]$ and [21].

\subsection{Case 1}

Consider the path of the thermo-mechanical loading cycle as shown in Figure 4 which was used in the experiments reported in $[19,20,41]$. This path consists of four processes. In the first process $(\mathrm{A} \rightarrow \mathrm{B})$, the SMPs were deformed by an external force under an invariable high temperature above its transition temperature $\left(T_{t}\right)$, and the stress and strain of SMPs were recorded. In the second process $(\mathrm{B} \rightarrow \mathrm{C})$, the displacements of SMPs were held and the temperature was lowered in a constant rate, the stress and temperature of the SMPs were 
recorded. In the third process $(C \rightarrow D)$, the external force was removed, the SMPs were fixed in the temporary shape and the strains of the temporary shape were recorded. In the last process $(\mathrm{D} \rightarrow \mathrm{A})$, the deformation was recovered freely with SMPs being reheated in a constant rate, the stress and strain of SMPs were recorded.

In the first process $(\mathrm{A} \rightarrow \mathrm{B})$, the thermal parts didn't exist due to that the testing temperature is constant. All of the frozen phase is in free state as the experimental environment of the SMPs is a constant high temperature. The modulus of the material is very small when the material is in free state. Thus, the mechanical properties of the active phase are considered as the mechanical properties of SMPs in this process. The relevant constitutive equation for the new model in Fig 3 is expressed as equation (1).

In the second process $(B \rightarrow C)$, the displacement of the SMPs is held constant and the test temperature is decreased from high to low temperature. The total strain of the SMPs is constant and the stress of the SMPs is changed with the reduction in temperature. In this process, the frozen phase started to transform from free state to frozen state, and the thermal expansion of SMPs played a role with the change in temperature. When the temperature was decreased, the strains of thermal parts decreased, and the strains of mechanical parts increased because the displacements of SMPs were held. The change of total stress of SMPs in this process is caused by the increase in strains of mechanical parts. Additionally, the transformation of frozen phase didn't finish instantaneously and this transformation process continues for some time. The thermal expansions of the active phase and frozen phase continue in the whole second process of the experiments. Thus the stress of frozen phase was dependent on the accumulation of the transformation ratio of frozen phase and the strains of the mechanical parts. The stress of the frozen phase was dependent on the pre-strain and the strain of the mechanical parts. At the same time, the active phase and frozen phase entered the stress relaxation process due to the tensile deformation.

By using the present model, the total stress can be expressed as follows:

$$
\sigma(\tilde{T})=\sigma_{a}(\tilde{T})+\sigma_{f}(\tilde{T})
$$

where $\sigma$ is the total stress of the system, $\tilde{T}$ is the testing temperature, and $\sigma_{a}$ and $\sigma_{f}$ denote respectively the stresses of the active and frozen phases to be determined.

The stress of active phase at the temperature of $\tilde{T}$ can be calculated using:

$$
\sigma_{a}(\tilde{T})=\varepsilon_{p r e} Y_{a}(\tilde{T})+\int_{T_{h}}^{\tilde{T}} \alpha Y_{a}(T) \mathrm{d} T
$$


where $\varepsilon_{\text {pre }}$ is the strain of the SMPs at the end of the first process, $\alpha$ is the coefficient of thermal expansion of the system (according to the test results, the coefficient of thermal expansion is constant, thus $\alpha_{T_{h}}=\alpha_{T_{l}}$ ), and $\tilde{T}$ is the testing temperature. The relaxation modulus of the active phase in equation (7) is given by

$$
Y_{a}(T)=Y_{a}\left(T_{h}-t_{e q u} \beta\right)
$$

where

$$
\begin{gathered}
Y_{a}(t)=\frac{\left[\left(q_{a 1}-A q_{a 2}\right) e^{-A t}-\left(q_{a 1}-B q_{a 2}\right) e^{-B t}\right]}{\sqrt{p_{a 1}{ }^{2}-4 p_{a 2}}} \\
A=\frac{p_{a 1}-\sqrt{p_{a 1}{ }^{2}-4 p_{a 2}}}{2 p_{a 2}}, \quad B=\frac{p_{a 1}+\sqrt{p_{a 1}{ }^{2}-4 p_{a 2}}}{2 p_{a 2}}
\end{gathered}
$$

and $t_{\text {equ }}$ is the equivalent time of the first process, $\beta$ is the cooling rate.

To determine the stress of the frozen phase, let us consider the constitutive equation of the frozen phase in frozen state:

$$
\sigma_{f}+p_{f 1} \dot{\sigma}_{f}=q_{f 0} \varepsilon_{f}+q_{f 1} \dot{\varepsilon}_{f}
$$

where $p_{f 1}=\frac{\mu_{f}}{E_{1 f}}, q_{f 0}=E_{2 f}$ and $q_{f 1}=\frac{\mu_{f}\left(E_{1 f}+E_{2 f}\right)}{E_{1 f}}$.

By solving equation (10), the stress of the frozen phase at temperature $\tilde{T}$ can be determined by:

$$
\sigma_{f}(\tilde{T})=\int_{T_{h}}^{\tilde{T}} \alpha Y_{f}(T) \mathrm{d} T
$$

where the relaxation modulus of the frozen phase at temperature $\tilde{T}$ is given by

$$
\begin{gathered}
Y_{f}(T)=\sum_{i=1}^{n}\left(\phi_{f}(T(i))-\phi_{f}(T(i-1))\right) Y_{f}\left(\left(T_{h}-T(i)\right) / \beta\right) \\
n=\left(T_{l}-T_{h}\right) / \Delta T, T(i)=T_{h}+i \Delta T,
\end{gathered}
$$

and the relaxation modulus of the frozen phase in frozen state can be determined from:

$$
Y_{f}(t)=q_{f 0}+\left(\frac{q_{f 1}}{p_{f 1}}-q_{f 0}\right) e^{-t / p_{f 1}}
$$

In the third process $(\mathrm{C} \rightarrow \mathrm{D})$, thermal expansions of the active phase and frozen phase have no effect due to the constant low temperature. Additionally, the relaxation process for the active phase and frozen phase in this process is neglected due to the low temperature.

The fixed strain of SMPs can be expressed as: 


$$
\varepsilon_{f i x}=\left(\varepsilon_{p r e}-\varepsilon_{r e s}\right) \frac{E_{1 a}+E_{2 a}}{E_{1 a}+E_{2 a}+E_{1 f}+E_{2 f}}
$$

where $\varepsilon_{r e s}$ is the irreversible strain of the SMPs. The irreversible deformation appeared in the experiments due to the low crosslinking degree, and it occurred in the first and second processes of the experiments.

In the first process, the irreversible strain $\varepsilon_{f i r}$ is given by:

$$
\varepsilon_{f i r}(t)=\int_{0}^{t_{1}} \frac{\sigma_{f i r}(t)}{u_{2 a}} \mathrm{dt} t=\varepsilon / \dot{\varepsilon}
$$

where $\sigma_{f i r}(t), \varepsilon, \dot{\varepsilon}, t$ is the stress, strain, strain rate and time of the active phase in the first process.

In the second process, the irreversible strain $\varepsilon_{\text {sec }}$ can be expressed as:

$$
\varepsilon_{\mathrm{sec}}(t)=\int_{0}^{t_{2}} \frac{\sigma_{\mathrm{sec}}(t)}{u_{2 a}} \mathrm{dt}, t=\left(T_{h}-T\right) / \beta
$$

where $\sigma_{\text {sec }}(t), T, \beta$ is the stress, temperature and temperature rate of the active phase in the second process.

The total irreversible strain $\varepsilon_{\text {res }}$ of the first two processes is then given by:

$$
\varepsilon_{\text {res }}=\varepsilon_{\text {fir }}+\varepsilon_{\mathrm{sec}}
$$

In the last process of the experiments $(\mathrm{D} \rightarrow \mathrm{A})$, the SMPs recovered their original shapes from the temporary shapes freely when reheated. In this process, the frozen phase started to transform from frozen state to free state with an increase in temperature. The stress was equal to zero due to the fact that the SMPs recovered to their shapes freely. The thermal expansion of SMPs has no effect on the total stress which is equal to the stress of mechanical parts in the present model. In addition, the relaxation process for the active and frozen phases is neglected because there is no external force applied in this process. Therefore the stresses of the active phase and the frozen phase can be expressed as follows:

$$
\sigma_{a}+\sigma_{f}=0
$$

In this process, the thermal expansion has no effect on the total stress. However, the strain caused by thermal expansion must be added to the total strain.

The total strain is given by:

$$
\varepsilon=\varepsilon_{m}+\varepsilon_{\text {res }}+\varepsilon_{T}
$$

where the thermal expansion induced strain can be calculated using: 


$$
\varepsilon_{T}=\alpha\left(T-T_{l}\right)
$$

The stresses of the active phase and the frozen phase can be respectively expressed as follows:

$$
\begin{gathered}
\sigma_{a}=\varepsilon_{m}\left(E_{1 a}+E_{2 a}\right) \\
\sigma_{f}=\phi_{f}\left(\varepsilon_{m}-\varepsilon_{f i x}\right)\left(E_{1 f}+E_{2 f}\right)
\end{gathered}
$$

\subsection{Case 2}

For case 2, Fig 5 depicts the path of the thermo-mechanical loading cycle used in the experiments in [21]. Similar to Case 1, this path has four processes. In the first process $(\mathrm{A} \rightarrow$ B), the SMPs were deformed by an external force under an invariable high temperature which is higher than the transform temperature of SMPs, the stresses and strains of SMPs were recorded. In the second process $(\mathrm{B} \rightarrow \mathrm{C})$, the displacements of SMPs were held and the temperature was reduced in a constant rate, the stresses and temperatures of SMPs were recorded. In the third process $(\mathrm{C} \rightarrow \mathrm{B})$, the displacements of SMPs were still held and the temperature increased in a constant rate, the stresses and temperatures of SMPs were recorded. In the last process $(\mathrm{B} \rightarrow \mathrm{A})$, the deformation recovered freely.

In the first process $(A \rightarrow B)$ and last process $(B \rightarrow A)$ in [21], the thermal parts were not exist due to the testing temperature is constant. The pre-strains $( \pm 9.1 \%)$ are considered as small deformations because they are less than $\pm 10 \%$ and this assumption is also existed in [21]. Thus the dashpots in the composite system don't play a role in the thermo-mechanical cycle experimentations. In these two processes, the relations of stresses and strains are almost proportional which conformed to Hooke's law.

Similar as the second process in $[19,20,41]$, the stresses of composite system are affected by mechanical parts and thermal parts together in the second process $(B \rightarrow C)$ in [21]. The stresses of SMPs are composed of the stresses of active phase and frozen phase. The stress of frozen phase is depended on the pre-strain and the strain of the mechanical parts, and the stress of frozen phase is depended on the accumulation of transformation ratio and the strains of the mechanical parts. However, the stress relaxation processes of two phases don't occurred in this process.

In this model, the total stress was expressed as:

$$
\sigma(\tilde{T})=\sigma_{a}(\tilde{T})+\sigma_{f}(\tilde{T})
$$


Where $\sigma$ is the total stress of the system, $\sigma_{a}$ is the stress of active phase, $\sigma_{f}$ is the stress of frozen phase, $\tilde{T}$ is the testing temperature.

The stress of active phase at the temperature of $\tilde{T}$ is expressed as:

$$
\sigma_{a}(\tilde{T})=\varepsilon_{p r e} E_{a}+\int_{T_{h}}^{\tilde{T}} \alpha_{c}(T) E_{a} \mathrm{~d} T, E_{a}=E_{1 a}+E_{2 a}
$$

Where $\varepsilon_{p r e}$ is the strain of SMPs in the last of the first process. $E_{a}$ is the equivalent modulus of active phase, $\alpha_{c}$ is the coefficient of thermal expansion in cooling process. $\tilde{T}$ is the testing temperature.

The equivalent modulus $E_{c f}$ of frozen phase at the temperature of $T$ in cooling process is expressed as:

$$
\begin{aligned}
& E_{c f}(T)=\sum_{i=1}^{n} E_{f}\left(\phi_{f}(T(i))-\phi_{f}(T(i-1))\right), \\
& n=\left(T_{l}-T_{h}\right) / \Delta T, T(i)=T_{h}+i \Delta T, E_{f}=E_{1 f}+E_{2 f}
\end{aligned}
$$

The stress of frozen phase at the temperature of $\tilde{T}$ was expressed as:

$$
\sigma_{f}(\tilde{T})=\int_{T_{h}}^{\tilde{T}} \alpha_{c}(T) E_{c f}(T) \mathrm{d} T
$$

In this process $(\mathrm{C} \rightarrow \mathrm{B})$, the frozen phase started to transform from frozen state to free state with the increment of temperature. The stresses of SMPs are composed of the stresses of active phase and frozen phase. The stress of frozen phase is depended on the pre-strain and the strain of the mechanical parts, and the stress of frozen phase is depended on the accumulation of transformation ratio and the strain of the mechanical parts. The stress relaxation processes of two phases don't occurred in this process. In this model, the total stress was expressed as:

$$
\sigma(\tilde{T})=\sigma_{a}(\tilde{T})+\sigma_{f}(\tilde{T})
$$

Where $\sigma$ is the total stress of the system, $\sigma_{a}$ is the stress of active phase, $\sigma_{f}$ is the stress of frozen phase, $\tilde{T}$ is the testing temperature.

The stress of active phase at the temperature of $\tilde{T}$ was expressed as:

$$
\sigma_{a}(\tilde{T})=\varepsilon_{p r e} E_{a}+\int_{T_{h}}^{T_{l}} \alpha_{c}(T) E_{a} \mathrm{~d} T-\int_{T_{l}}^{\tilde{T}} \alpha_{h}(T) E_{a} \mathrm{~d} T
$$

Where $\alpha_{h}$ is the coefficient of thermal expansion in heating process.

The equivalent modulus $E_{h f}$ of frozen phase at the temperature of $T$ was expressed as: 


$$
\begin{aligned}
E_{h f}(T) & =\sum_{i=1}^{n} E_{f}\left(\phi_{f}(T(i))-\phi_{f}(T(i-1))\right) \\
n & =\left(T_{h}-T_{l}\right) / \Delta T, T(i)=T_{h}+i \Delta T,
\end{aligned}
$$

The stress of frozen phase at the temperature of $\tilde{T}$ was expressed as:

$$
\sigma_{f}(\tilde{T})=\int_{T_{h}}^{T_{l}} \alpha_{c}(T) E_{c f}(T) \mathrm{d} T-\int_{T_{l}}^{\tilde{T}} \alpha_{h}(T) E_{h f}(T) \mathrm{d} T
$$

\section{Results and Discussion}

In this section, numerical results are obtained using the detailed model formulations presented in Section 3 and are then compared with the calculated and measured results reported in $[19,20]$ and $[21]$.

\subsection{Comparison with the results in $[19,20]$.}

Table 1 presents the coefficients used in all calculation.

Fig 6 shows the elastic modulus-temperature curves obtained by Tobushi's experiment, Tobushi's calculation and the calculation of the developed model. The elastic modulus E of the new model is express as:

$$
E=\left(E_{1 a}+E_{2 a}\right)+\phi_{f}\left(E_{1 f}+E_{2 f}\right)
$$

In this calculated process, the delay time $\tau$ was set as zero, and the cooling/heating rate $\beta$ was set as $1 \mathrm{~K} / \mathrm{min}$. As seen in Fig 6 , the present predicting elastic modulus-temperature curve isn't an ideally straight line. The elastic modulus is depend on the transformation of frozen phase, and the transform process is cause by the changes of temperature. The values and trend of the present predicting elastic modulus-temperature curve are close to the experimental results, thus transform equation and the transform process of frozen phase which is adopted from the process of crystallization are suitable.

Fig 7 shows the stress-strain curves obtained by Tobushi's experiment, Tobushi's calculation and the calculation of the developed model. The experimental and calculated results are based on the different pre-strain constraint conditions, and the pre-strains are $2.4 \%$, 4\%, $10 \%$ and 20\%, respectively. As seen in Fig 7, the stresses increase nonlinearly with the increment of strains in the first process $(\mathrm{A} \rightarrow \mathrm{B})$, this is due to the creep was occurred in SMPs which is presented by the dashpots in the developed model. In the second process $(B \rightarrow C)$, the stresses increase with the decrement of temperature. At the end of third process $(C \rightarrow D)$, the SMPs were fixed in the temporary shape, this is an important characteristic of SMPs. The 
present predicting stress-strain curves express the inclination of Tobushi's experimental and calculated results.

Fig 8 shows the stress-temperature curves obtained by Tobushi's experimentation, Tobushi's calculation and the calculation of the developed model. A special change of stresstemperature curves occurred in the second process $(B \rightarrow C)$. The total stress of SMPs is changed with the decrement of temperature, however the total strain of SMPs is constant. This is due to the thermal expansion and the transform of phase in SMPs. In the developed model, this process is expressed clearly. The mechanical parts of the model expressed the relationship of stress and mechanical strain given in SMPs and the transform of frozen phase. The thermal parts of the model expressed the relationship of temperature and thermal strain given in SMPs. The mechanical strain and thermal strain are used to strung the two parts together because the total strain was constant. The stress of frozen phase was depended on the accumulation of transformation ratio and the strain of the mechanical parts. The stress of frozen phase was depended on the pre-strain and the strain of the mechanical parts. The stresses of SMPs were equal to the sum of the stresses of active phase and frozen phase. Additionally, the stress relaxation processes of active phase and frozen phase in the first and second processes are considered. As seen in Fig 8, the developed model has a nice description to Tobushi's experimental results.

Fig 9 shows the strain-temperature curves obtained by Tobushi's experimentation, Tobushi's calculation and the calculation of the developed model. At the end of third process $(\mathrm{C} \rightarrow \mathrm{D})$, the SMPs were fixed in the temporary shapes. The fixed strains are not equal the prestrains. The gaps between fixed strains and pre-strains increase with the increment of prestrains. In the present model, this is due to the deformation for maintaining the equilibrium state of mechanical parts and the irreversible deformation occurred in the first and second processes. The last process $(\mathrm{D} \rightarrow \mathrm{A})$ is similar to the second process except the total stress is zero. In this process, the frozen phase transforms from frozen state to free state and none external force exist in the whole process. There is a relationship among the stresses and mechanical strains of active phase and frozen phase due to the mechanical parts of the model are maintained in the equilibrium state. Additionally, thermal strain and the irreversible deformation are added to the total strain. As seen in Fig 9, the present predicting results are consonant with Tobushi's experimental results preferably.

\subsection{Comparison with the results in [21]}

Table 2 presents the coefficients used in all calculation. 
Fig 10 shows the thermal expansion strain-temperature curves obtained by Liu's experimentation and the calculation of the developed model. The thermal expansion coefficient of the system was expressed by equation (5). In this calculated process, the delay time $\tau$ was set as zero, and the cooling/heating rate $\beta$ was set as $1 \mathrm{~K} / \mathrm{min}$. As seen in Fig 11, the thermal expansion coefficients decrease with the decrement of temperature. In the present model, the change of thermal expansion of the composite system is depended on the transform of frozen phase between free state and frozen state. The present calculated results are close to Liu's experimental results. Thus the predicting transform process of frozen phase and the transform equations (4) and (5) are suitable.

Fig 11 and Fig 12 show the stress-temperature curves obtained by Liu's experiment, Liu's calculation and the calculation of the developed model in cooling and heating process, respectively. The stresses of SMPs are composed of the stresses of active phase and frozen phase and the stress of frozen phase is depended on the pre-strain and the strain of the mechanical parts. As seen in Fig 11 and Fig 12, the new model has a nice prediction and description to the experimental results in [21].

\section{Conclusions}

In the present study, a thermodynamics viscoelastic constitutive model was developed to describe the typical thermo-mechanical cycle experimentations. In this model, the changes of SMPs in thermodynamic and mechanics in cooling/heating process were described clearly and the irreversible deformations in the thermo-mechanical cycle experimentations were calculated according the new model.

Based on microstructure of semi-crystalline SMPs and the practical experimentations, a new type of microstructure of SMPs was proposed and new definitions of phases: active phase and frozen phase were set forth in the present study. The new thermodynamics viscoelastic model is series by two parts: mechanical parts and thermal parts. In view of viscoelasticity theory of polymer, a four elements structure and a three element structure was adopted to express the active phase and frozen phase respectively, and two one element structures represent thermal expansions of SMPs in high and low temperature in the developed model. According to the crystallization theory of polymer and the study of Wang, a new transform equation for the transformation of frozen phase was developed, and the transform delay time and cooling/heating rate were considered in this transform equation. In the developed model, the thermal expansion of SMPs is depended on the transform of frozen phase, and an equation 
based on the transformation of frozen phase was proposed to describe the change of thermal expansion coefficient of SMPs.

Finally, the typical thermo-mechanical cycle experimentation processes were calculated using the new thermodynamics viscoelastic constitutive model. Through the comparison between the calculated results and Tobushi and Liu's experimental results, the rationality and accuracy of the new thermodynamics viscoelastic constitutive model was finally verified.

\section{Acknowledgements}

The authors wish to acknowledge the financial support by the National Science Foundation of China (11472086), the Ph.D. Programs Foundation of Ministry of Education of China (20112304110015) and the Australian Research Council via a Discovery Grant project (DP130103958).

\section{References}

[1] Chen S, Hu J, Zhuo H. Properties and mechanism of two-way shape memory polyurethane composites. Composites Science and Technology. 2010;70(10):1437-43.

[2] Ratna D, Karger-Kocsis J. Recent advances in shape memory polymers and composites: a review. Journal of Materials Science. 2008;43(1):254-69.

[3] Behl M, Lendlein A. Shape-memory polymers. Materials today. 2007;10(4):20-8.

[4] Liu C, Qin H, Mather P. Review of progress in shape-memory polymers. Journal of Materials Chemistry. 2007;17(16):1543-58.

[5] Mather PT, Luo X, Rousseau IA. Shape memory polymer research. Annual Review of Materials Research. 2009;39:445-71.

[6] Sahoo NG, Jung YC, Yoo HJ, Cho JW. Influence of carbon nanotubes and polypyrrole on the thermal, mechanical and electroactive shape-memory properties of polyurethane nanocomposites. Composites Science and Technology. 2007;67(9):1920-9.

[7] Guo J, Wang Z, Tong L, Lv H, Liang W. Shape memory and thermo-mechanical properties of shape memory polymer/carbon fiber composites. Composites Part A: Applied Science and Manufacturing. 2015;76:162-71.

[8] Liu Y, Lv H, Lan X, Leng J, Du S. Review of electro-active shape-memory polymer composite. Composites Science and Technology. 2009;69(13):2064-8.

[9] Uchino K. Shape memory ceramics. Shape Memory Materials. 1999:184-202.

[10] Otsuka K, Ren X. Recent developments in the research of shape memory alloys. Intermetallics. 1999;7(5):511-28.

[11] Hu JL, Ji FL, Wong YW. Dependency of the shape memory properties of a polyurethane upon thermomechanical cyclic conditions. Polymer international. 2005;54(3):600-5.

[12] Lan X, Liu Y, Lv H, Wang X, Leng J, Du S. Fiber reinforced shape-memory polymer composite and its application in a deployable hinge. Smart Materials and Structures. 2009;18(2):024002.

[13] Li H, Zhong J, Meng J, Xian G. The reinforcement efficiency of carbon nanotubes/shape memory polymer nanocomposites. Composites Part B: Engineering. 2013;44(1):508-16.

[14] Meng Q, Hu J. A review of shape memory polymer composites and blends. Composites Part A: Applied Science and Manufacturing. 2009;40(11):1661-72. 
[15] Wang CC, Huang WM, Ding Z, Zhao Y, Purnawali H. Cooling-/water-responsive shape memory hybrids. Composites Science and Technology. 2012;72(10):1178-82.

[16] You Z. Folding structures out of flat materials. Science. 2014;345(6197):623-4.

[17] Felton SM, Tolley MT, Onal CD, Rus D, Wood RJ. Robot self-assembly by folding: A printed inchworm robot. Robotics and Automation (ICRA), 2013 IEEE International Conference on: IEEE; 2013. p. 277-82.

[18] Felton S, Tolley M, Demaine E, Rus D, Wood R. A method for building self-folding machines. Science. 2014;345(6197):644-6.

[19] Tobushi H, Hashimoto T, Hayashi S, Yamada E. Thermomechanical constitutive modeling in shape memory polymer of polyurethane series. Journal of Intelligent Material Systems and Structures. 1997;8(8):711-8.

[20] Tobushi H, Okumura K, Hayashi S, Ito N. Thermomechanical constitutive model of shape memory polymer. Mechanics of materials. 2001;33(10):545-54.

[21] Liu Y, Gall K, Dunn ML, Greenberg AR, Diani J. Thermomechanics of shape memory polymers: uniaxial experiments and constitutive modeling. International Journal of Plasticity. 2006;22(2):279-313.

[22] Chen Y-C, Lagoudas DC. A constitutive theory for shape memory polymers. Part I: Large deformations. Journal of the Mechanics and Physics of Solids. 2008;56(5):1752-65.

[23] Chen Y-C, Lagoudas DC. A constitutive theory for shape memory polymers. Part II: a linearized model for small deformations. Journal of the Mechanics and Physics of Solids. 2008;56(5):1766-78.

[24] Behl M, Lendlein A. Actively moving polymers. Soft Matter. 2007;3(1):58-67.

[25] Wang Z, Li D, Xiong Z, Chang R. Modeling thermomechanical behaviors of shape memory polymer. Journal of applied polymer science. 2009;113(1):651-6.

[26] Zhou B, Liu Y, Leng J. A macro-mechanical constitutive model for shape memory polymer. Science China Physics, Mechanics and Astronomy. 2010;53(12):2266-73.

[27] Zhou B, Liu Y-J, Lan X, Leng J-S, Yoon S-H. A glass transition model for shape memory polymer and its composite. International Journal of Modern Physics B. 2009;23(06n07):124853.

[28] Baghani M, Naghdabadi R, Arghavani J, Sohrabpour S. A thermodynamically-consistent 3D constitutive model for shape memory polymers. International Journal of Plasticity. 2012;35:13-30.

[29] Guo X, Liu L, Liu Y, Zhou B, Leng J. Constitutive model for a stress-and thermal-induced phase transition in a shape memory polymer. Smart Materials and Structures. 2014;23(10):105019.

[30] Guo X, Zhou B, Liu L, Liu Y, Leng J. A stress-induced phase transition model for semicrystallize shape memory polymer. SPIE Smart Structures and Materials+ Nondestructive Evaluation and Health Monitoring: International Society for Optics and Photonics; 2014. p. 905817--8.

[31] Bhattacharyya A, Tobushi H. Analysis of the isothermal mechanical response of a shape memory polymer rheological model. Polymer Engineering \& Science. 2000;40(12):2498-510.

[32] $\mathrm{Li} \mathrm{G}, \mathrm{Xu} \mathrm{W}$. Thermomechanical behavior of thermoset shape memory polymer programmed by cold-compression: testing and constitutive modeling. Journal of the Mechanics and Physics of Solids. 2011;59(6):1231-50.

[33] Nguyen TD, Qi HJ, Castro F, Long KN. A thermoviscoelastic model for amorphous shape memory polymers: incorporating structural and stress relaxation. Journal of the Mechanics and Physics of Solids. 2008;56(9):2792-814.

[34] Westbrook KK, Kao PH, Castro F, Ding Y, Qi HJ. A 3D finite deformation constitutive model for amorphous shape memory polymers: a multi-branch modeling approach for nonequilibrium relaxation processes. Mechanics of Materials. 2011;43(12):853-69. 
[35] Yu K, Xie T, Leng J, Ding Y, Qi HJ. Mechanisms of multi-shape memory effects and associated energy release in shape memory polymers. Soft Matter. 2012;8(20):5687-95.

[36] Khonakdar HA, Jafari SH, Rasouli S, Morshedian J, Abedini H. Investigation and modeling of temperature dependence recovery behavior of shape-memory crosslinked polyethylene. Macromolecular theory and simulations. 2007;16(1):43-52.

[37] Tsukada G, Tokuda M, Torii M. Temperature Triggered Shape Memory Effect of Transpolyisoprene-based Polymer. Journal of endodontics. 2014;40(10):1658-62.

[38] Choy C, Chen F, Ong E. Anistropic thermal expansion of oriented crystalline polymers. Polymer. 1979;20(10):1191-8.

[39] Davis G, Eby R, Colson J. Thermal expansion of polyethylene unit cell: effect of lamella thickness. Journal of Applied Physics. 1970;41(11):4316-26.

[40] Mason P. Thermal expansion and viscoelasticity of rubber in relation to crosslinking and molecular packing. Polymer. 1964;5:625-35.

[41] Tobushi H, Hara H, Yamada E, Hayashi S. Thermomechanical properties in a thin film of shape memory polymer of polyurethane series. 1996 Symposium on Smart Structures and Materials: International Society for Optics and Photonics; 1996. p. 46-57.

[42] Lendlein A, Kelch S. Shape-memory polymers. Angewandte Chemie International Edition. 2002;41(12):2034-57.

[43] Bhattacharyya A. Isothermal Mechanical Response of Shape Memory Polymer (SMP)Based Hybrid Models and SMP-Composites. Journal of the Mechanical Behavior of Materials. 2004;15(1-2):107-34.

[44] Lin Y-H. Polymer viscoelasticity: basics, molecular theories, experiments, and simulations: World Scientific; 2011.

[45] Rubinstein M, Colby RH. Polymer physics: OUP Oxford; 2003.

[46] Shaw MT, MacKnight WJ. Introduction to polymer viscoelasticity: John Wiley \& Sons; 2005.

[47] Avrami M. Transformation-time relations for random distribution of nuclei. J Chem Phys. 1940;8:212-24.

[48] Ozawa T. Kinetics of non-isothermal crystallization. Polymer. 1971;12(3):150-8.

Table 1 Values of the coefficients used

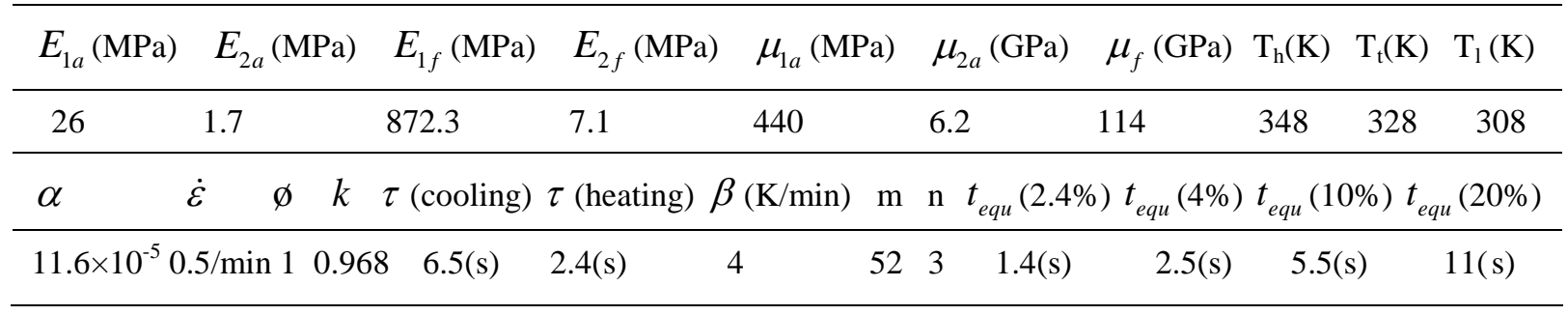

Table 2 Values of the coefficients used

\begin{tabular}{cllllll}
\hline$E_{a}(\mathrm{MPa})$ & $E_{f}(\mathrm{MPa})$ & $\mathrm{T}_{\mathrm{h}}(\mathrm{K})$ & $\mathrm{T}_{\mathrm{t}}(\mathrm{K})$ & $\mathrm{T}_{\mathrm{l}}(\mathrm{K})$ & $\alpha_{T_{h}}(1 / \mathrm{K})$ & $\alpha_{T_{l}}(1 / \mathrm{K})$ \\
\hline 8.8 & 741.2 & 358 & 343 & 273 & $1.8 \times 10^{-4}$ & $0.9 \times 10^{-}$ \\
\hline$\varnothing$ & $k$ & $\tau$ (cooling) & $\tau$ (heating) & $\beta(\mathrm{K} / \mathrm{min})$ & $\mathrm{m}$ & $\mathrm{n}$ \\
\hline 1 & 0.895 & $0.7(\mathrm{~s})$ & $2.5(\mathrm{~s})$ & 1 & 48 & 3 \\
\hline
\end{tabular}




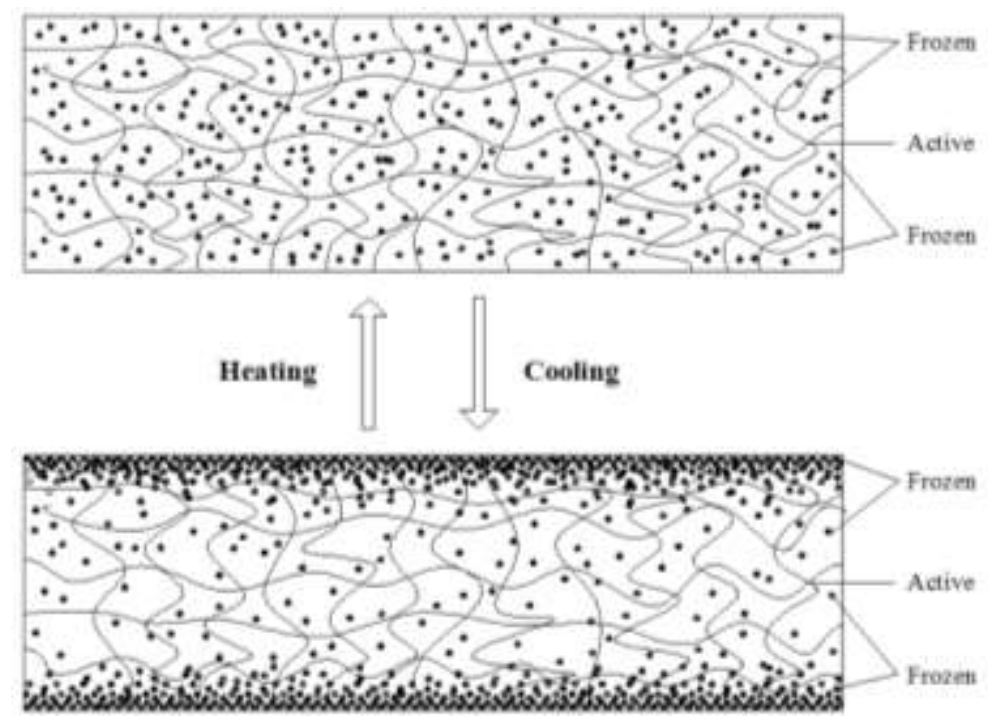

Fig 1 Schematic diagram of the microstructure of SMPs with a two-phase composite structure

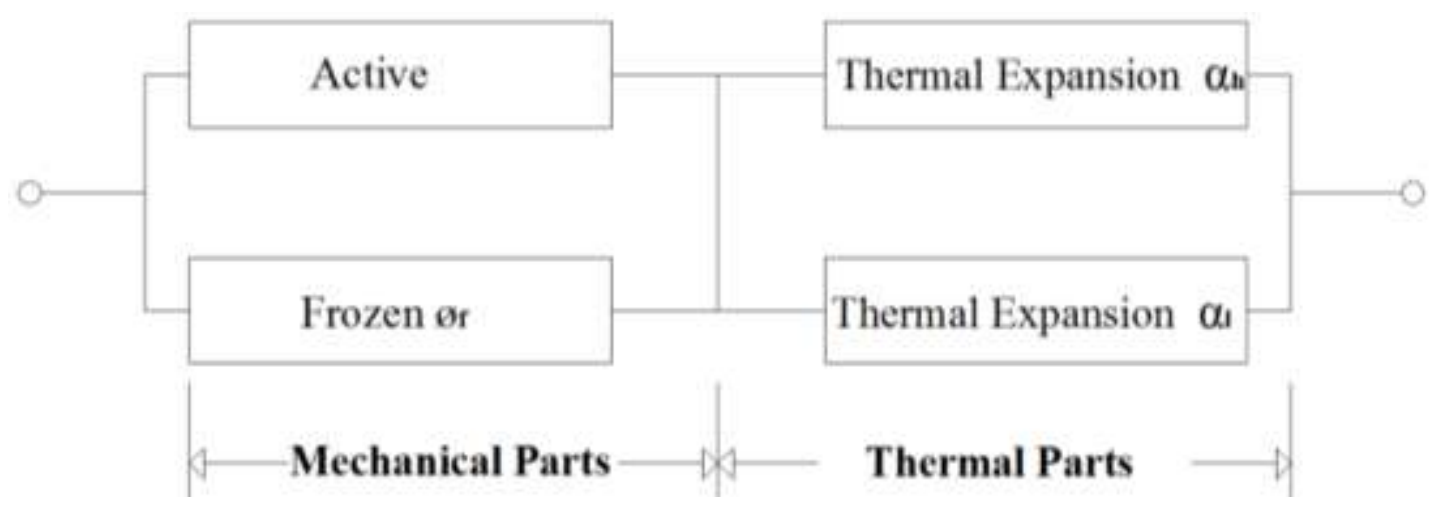

Fig 2 A schematic diagram of the thermodynamics constitutive model 


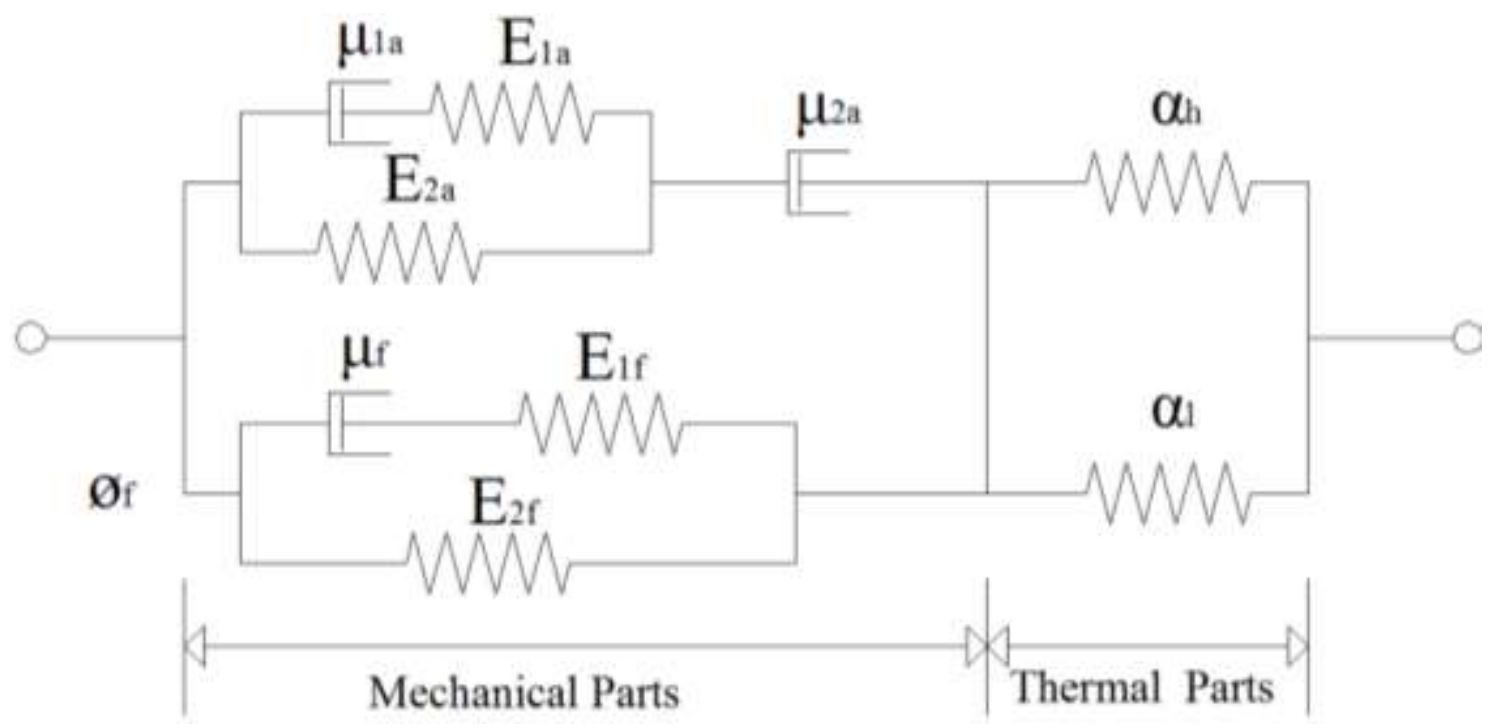

Fig 3 A schematic diagram of the thermodynamics viscoelastic constitutive model

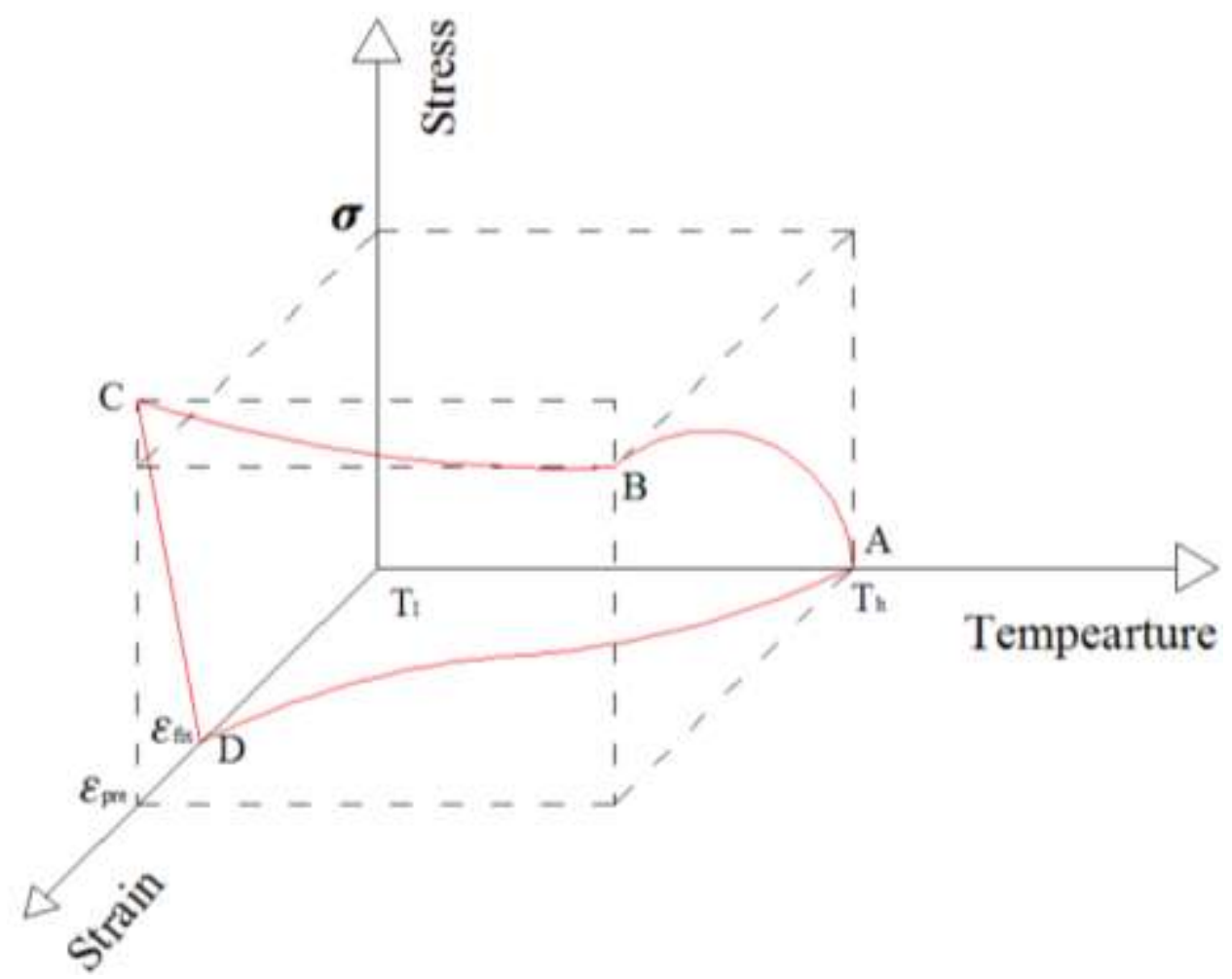

Fig 4 The path of the thermo-mechanical cycle used in the experiments $[19,20,41]$ 


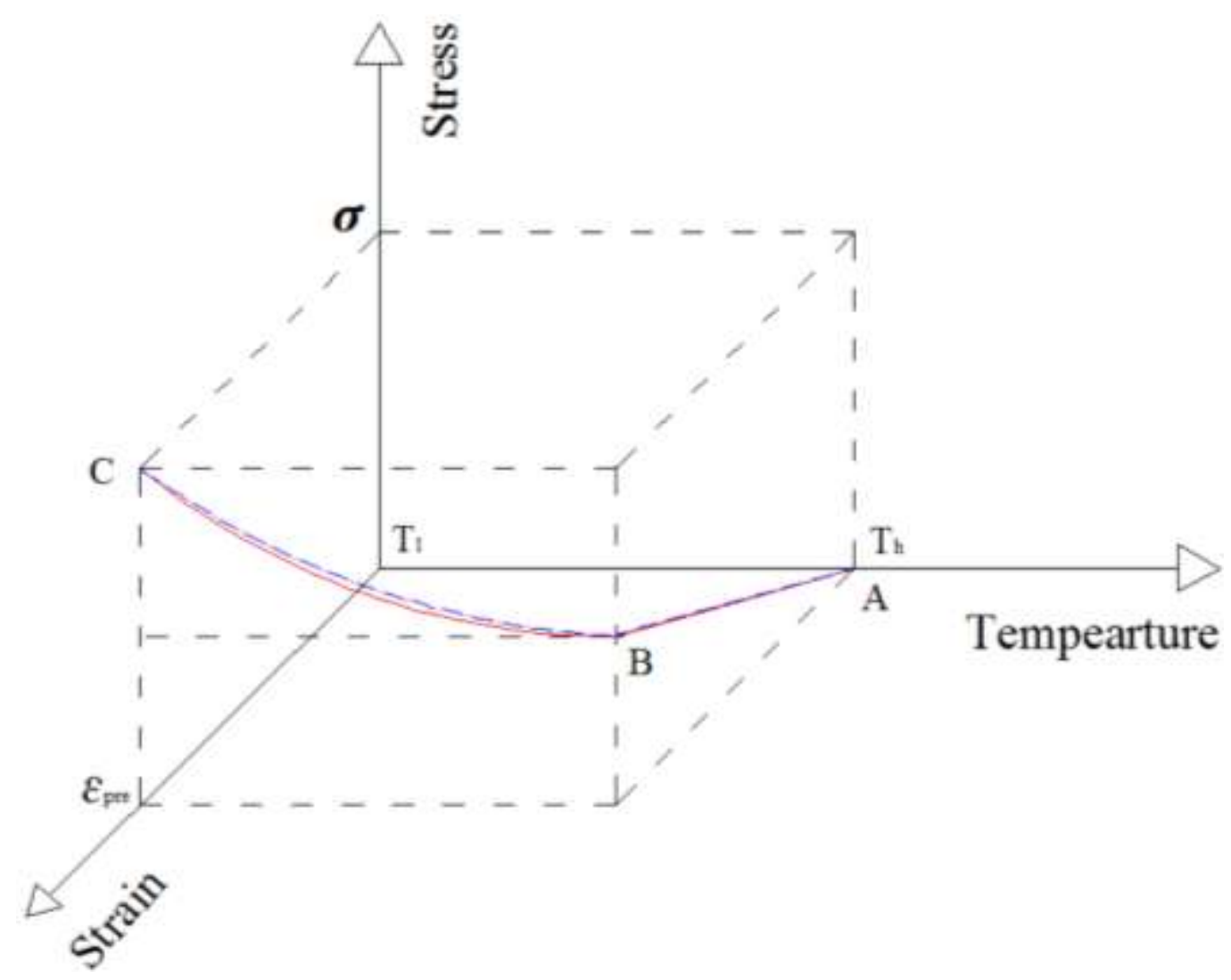

Fig 5 The path of the thermo-mechanical cycle experimentation in Liu's study [21].

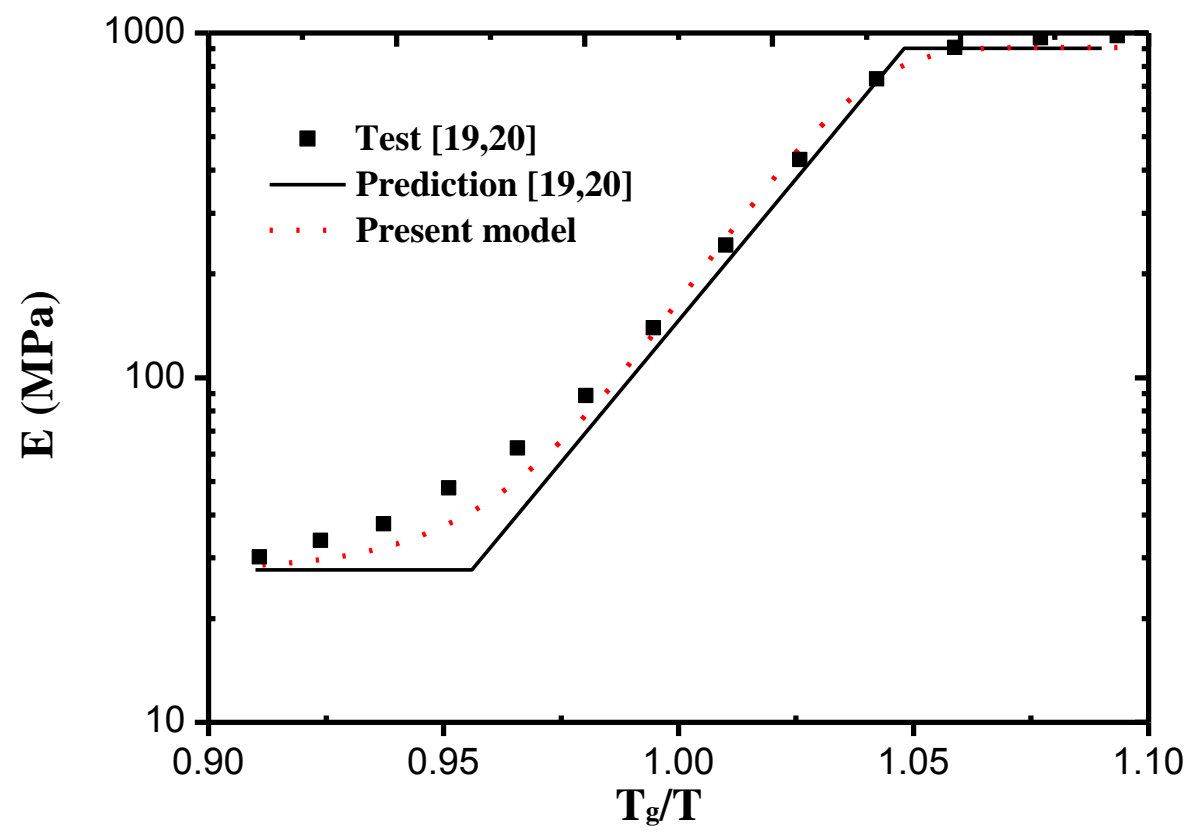

Fig 6 The elastic modulus-temperature curves obtained by Tobushi's experiment, calculation and the calculation of the new model. 


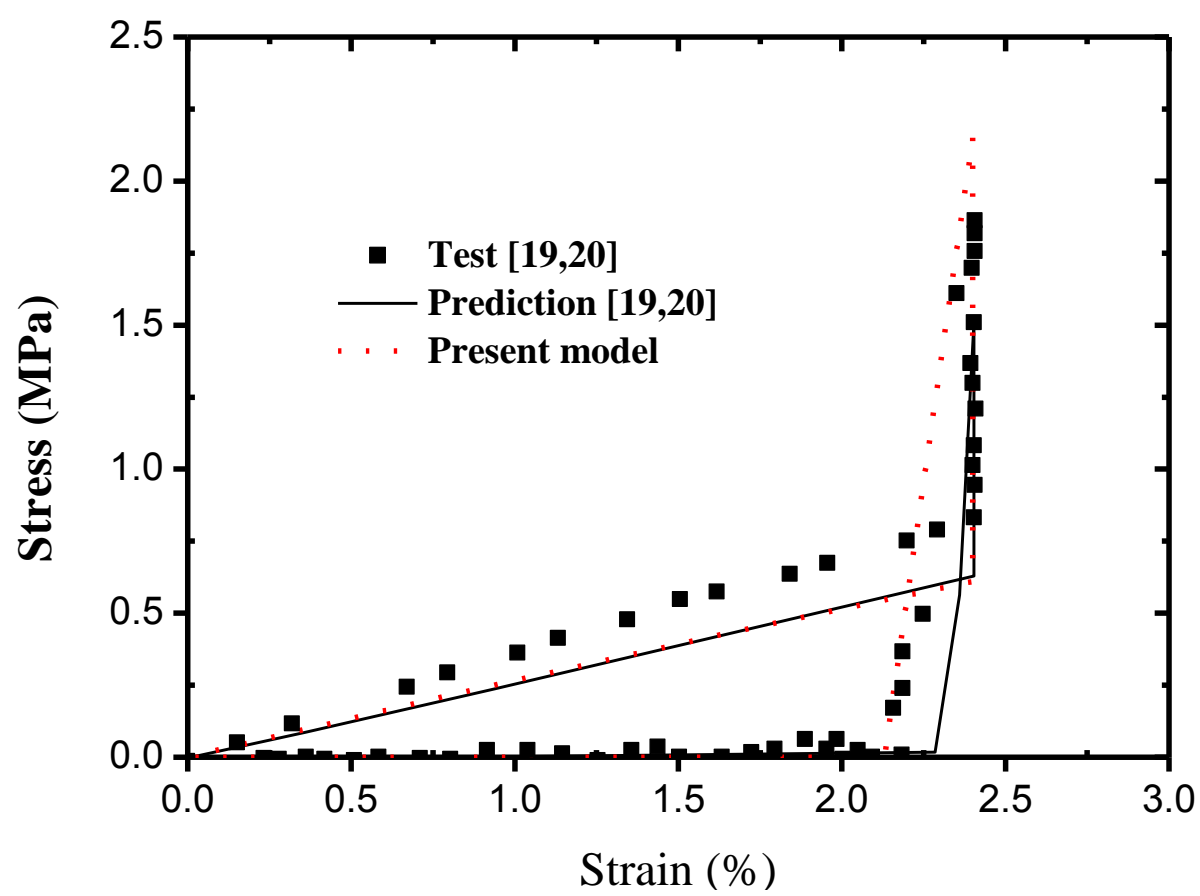

(a)

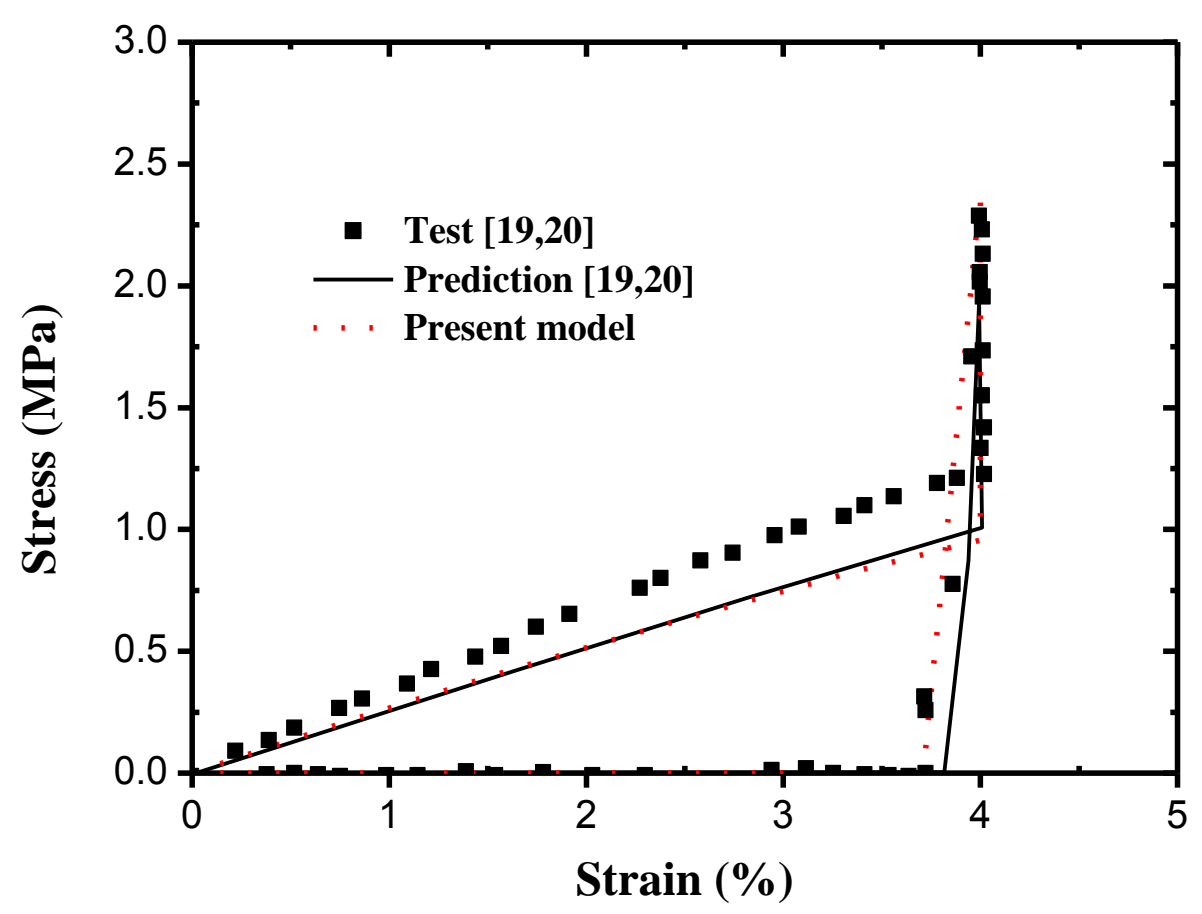

(b) 


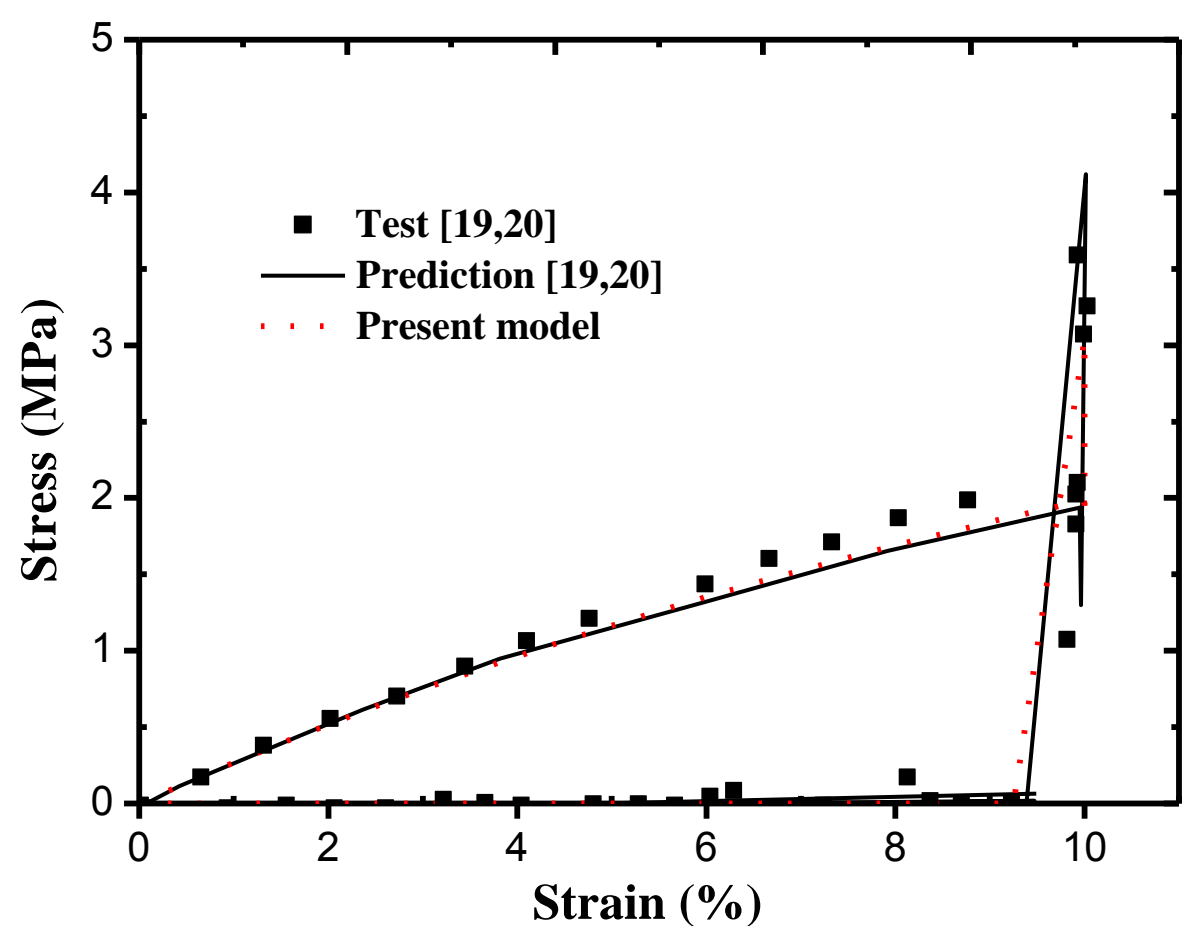

(c)

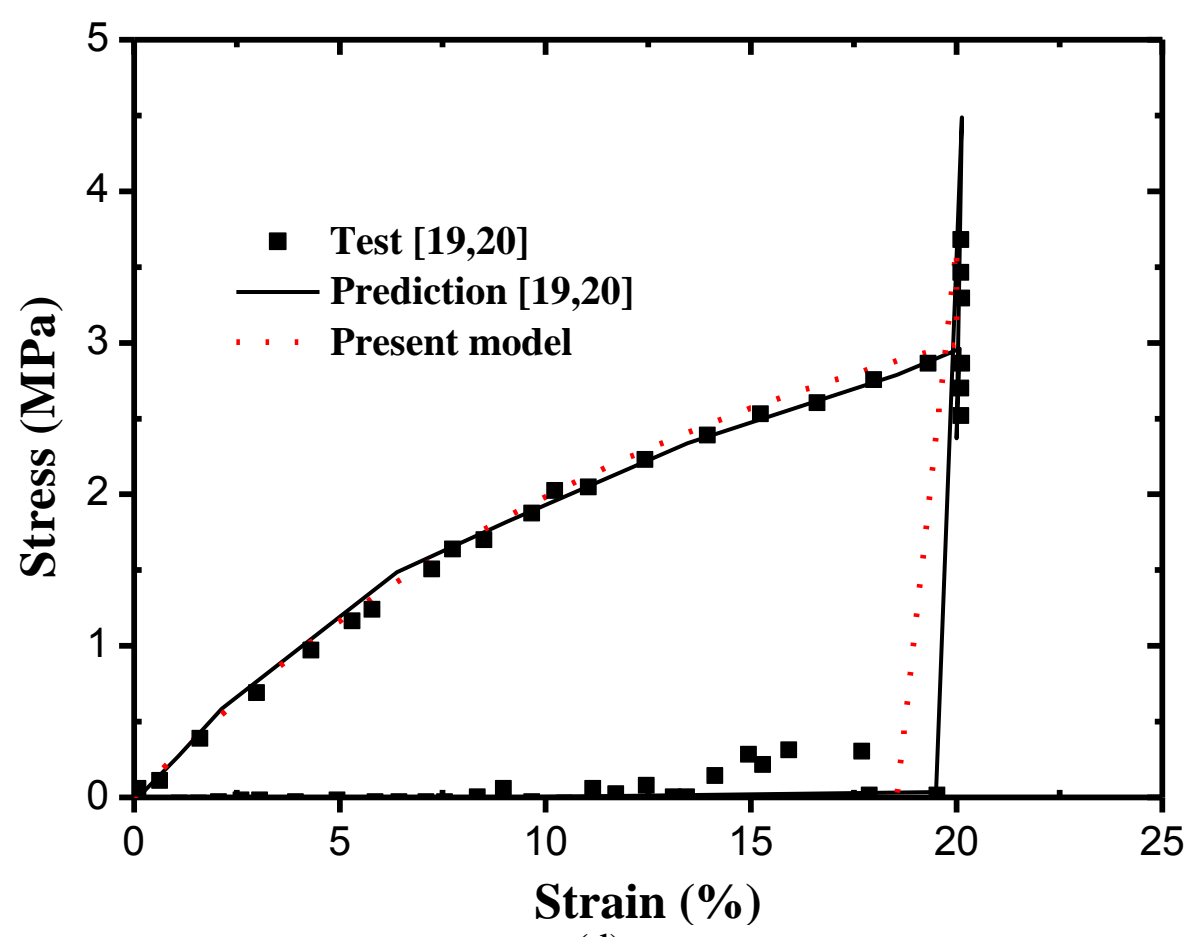

(d)

Fig 7(a)-(d) The stress-strain curves obtained by Tobushi's experiment, calculation and the calculation of the new model. 


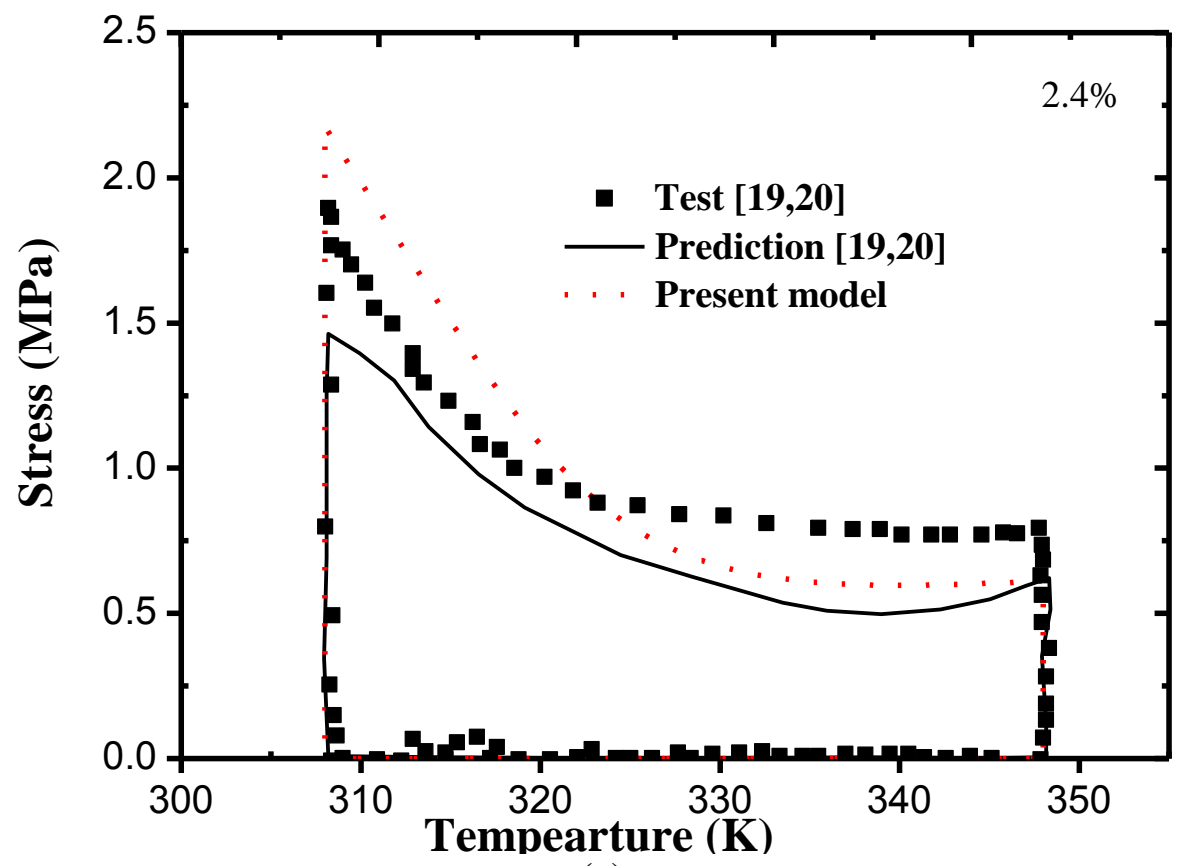

(a)

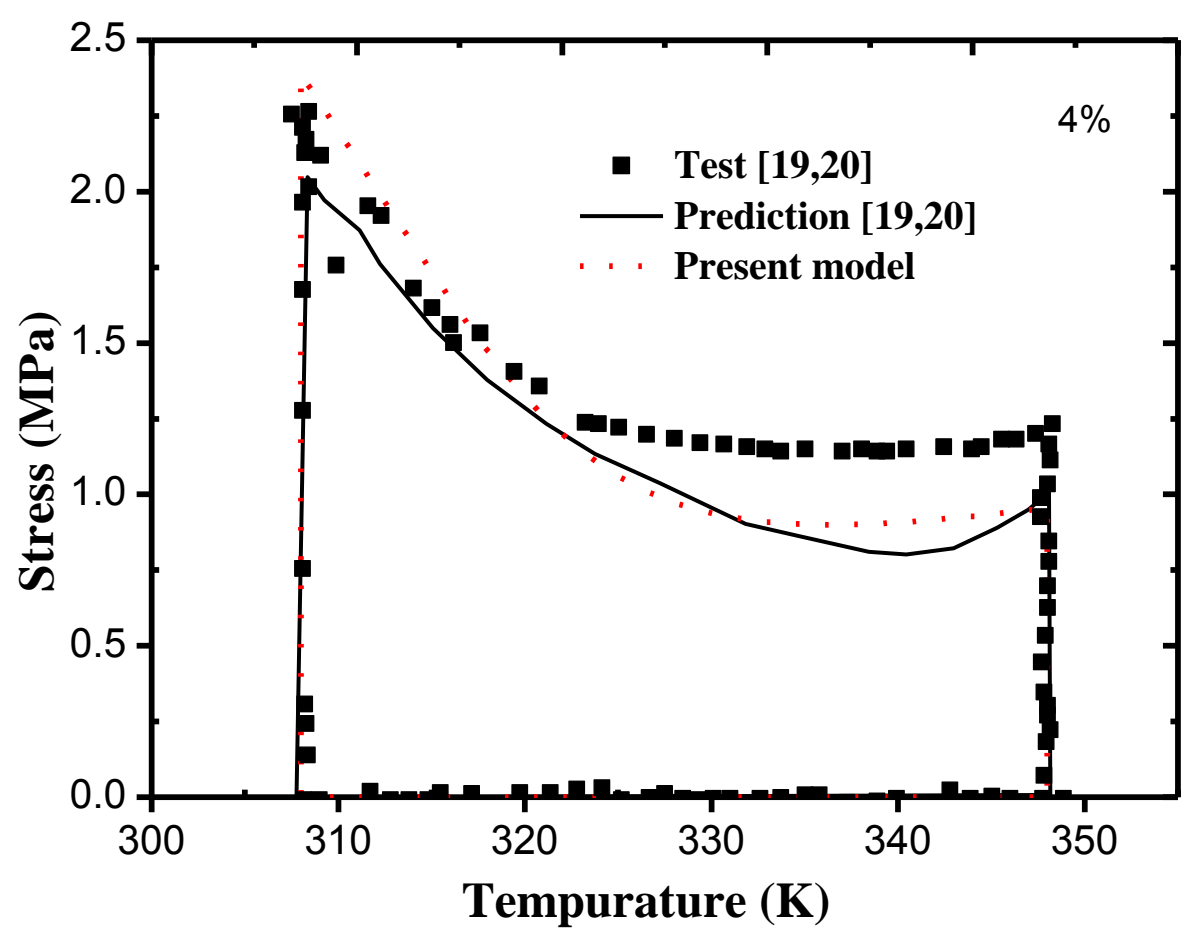

(b) 


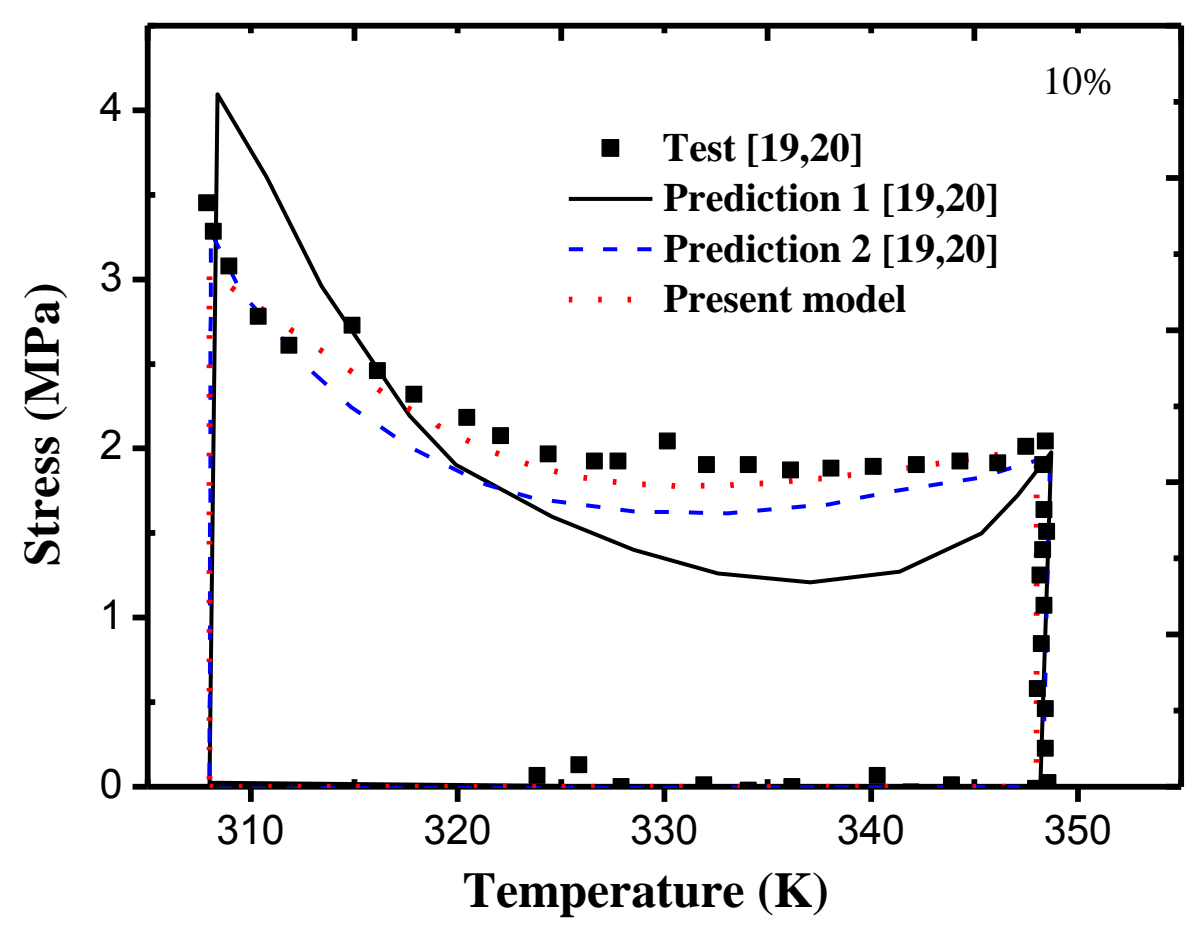

(c)

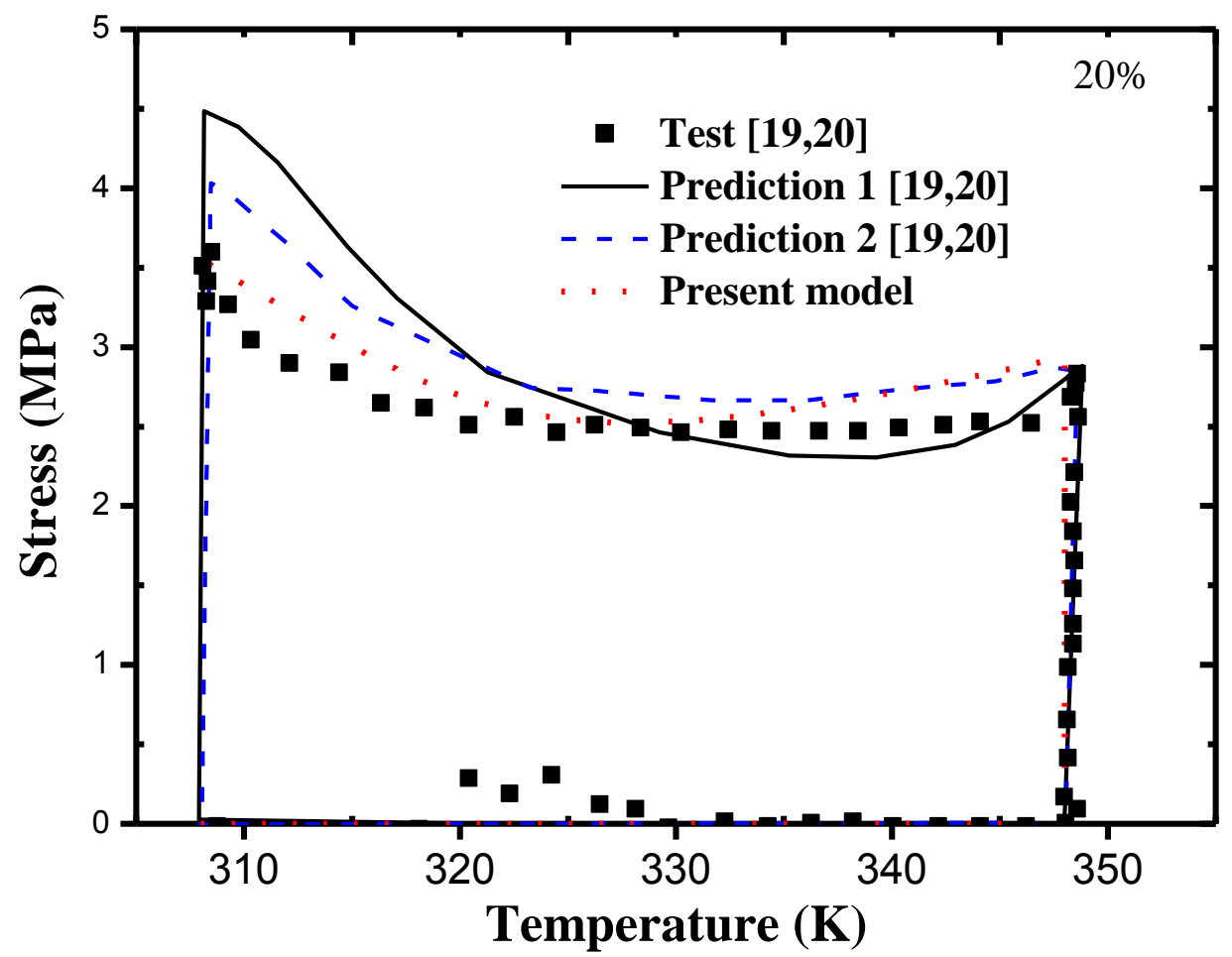

(d)

Fig 8. The stress-temperature curves obtained by Tobushi's experimentation, calculation and the calculation of the new model. 


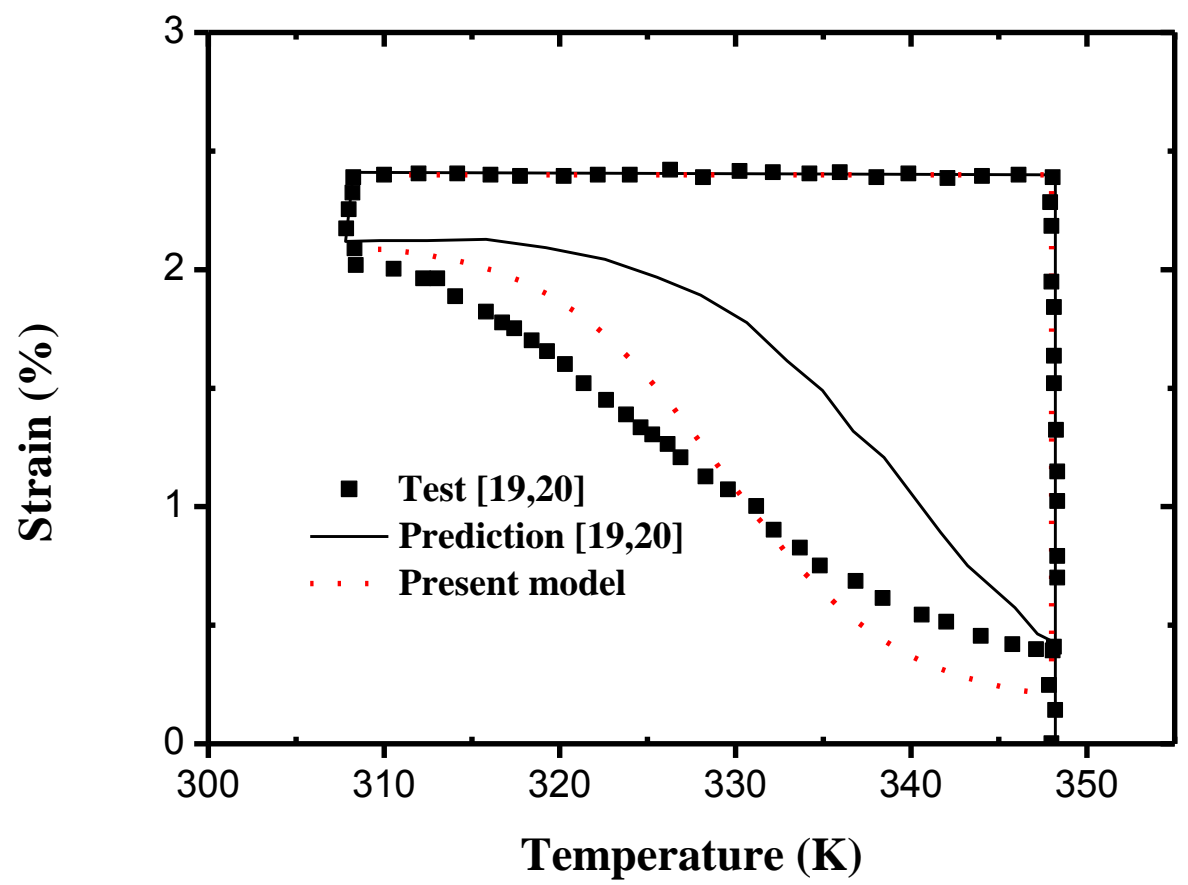

(a)

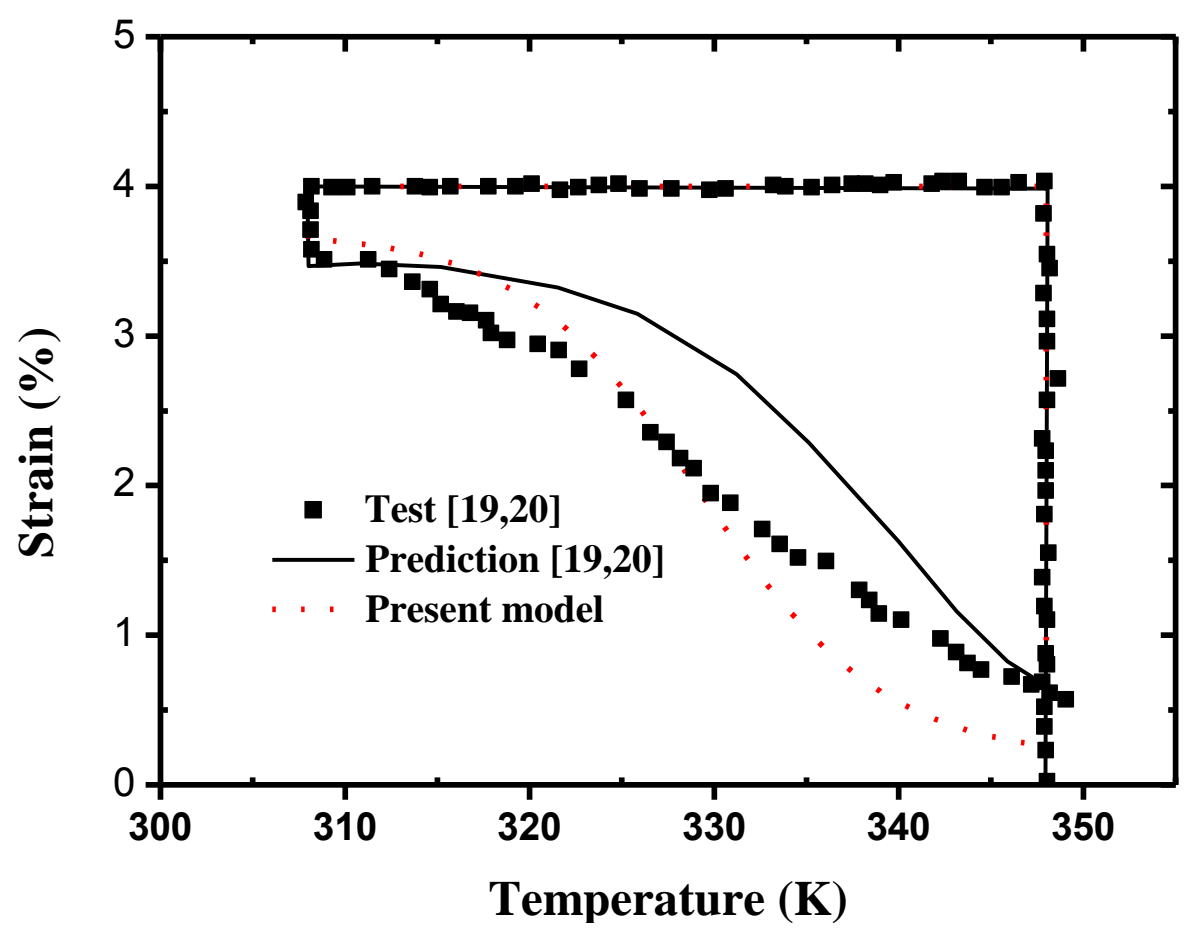

(b) 


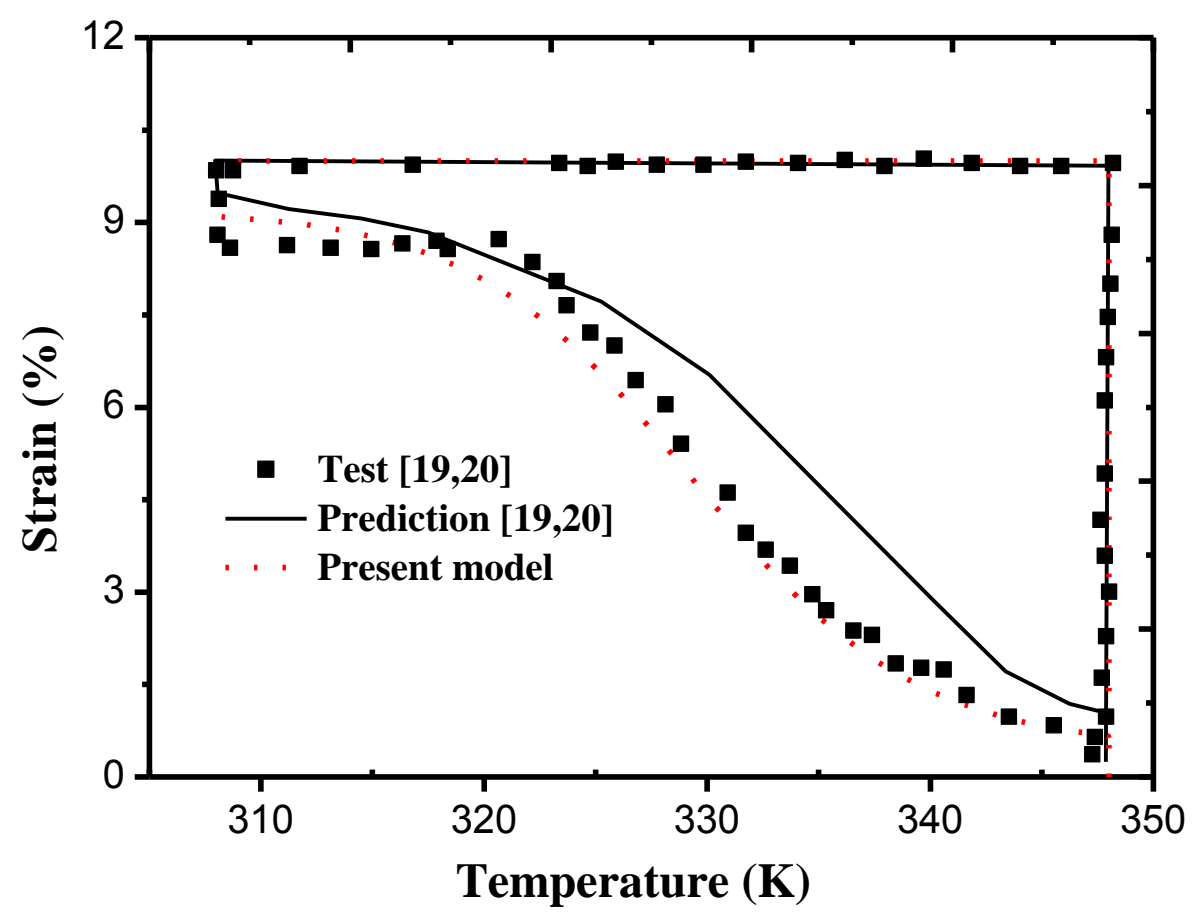

(c)

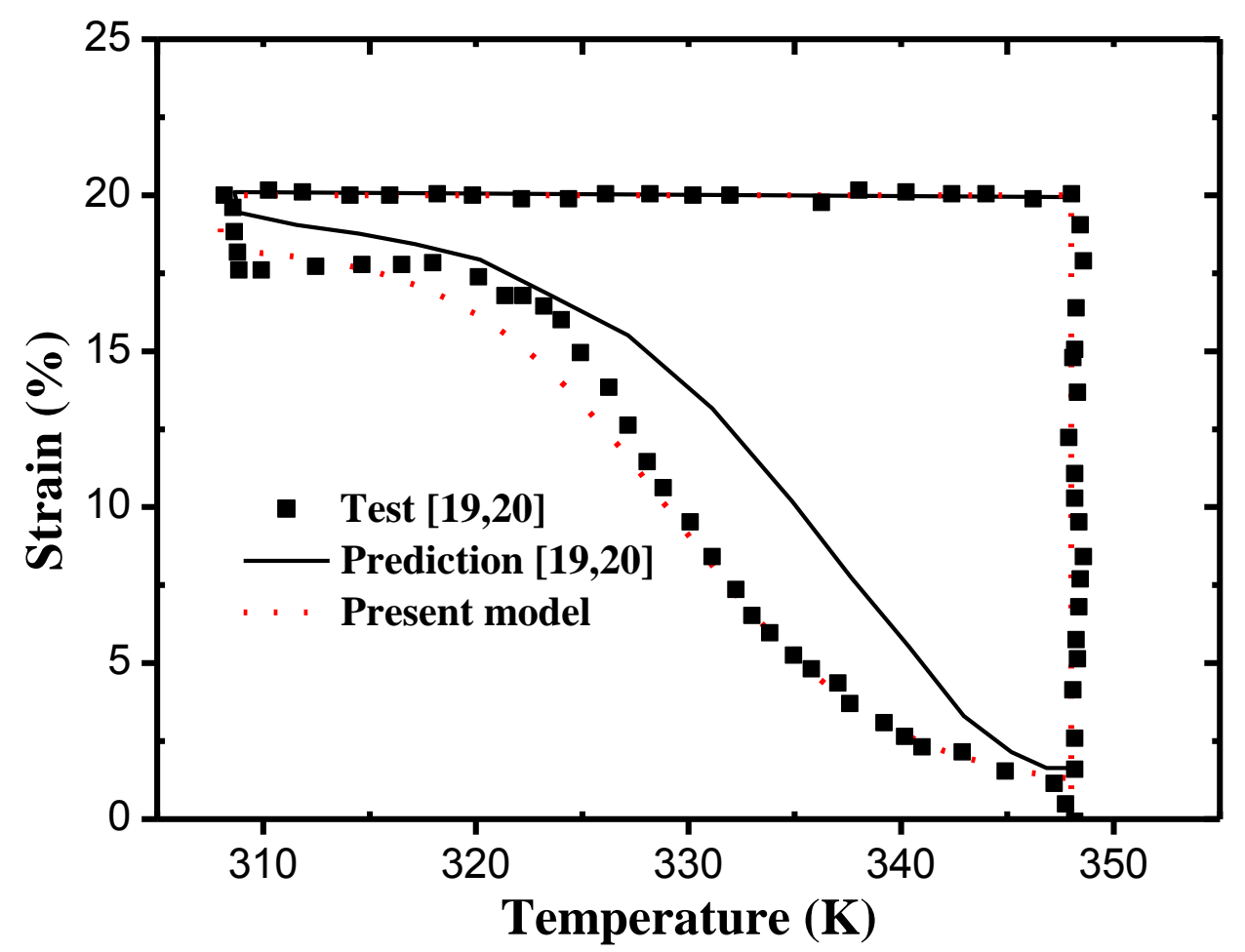

(d)

Fig 9 The strain-temperature curves obtained by Tobushi's experimentation, calculation and the calculation of the new model. 


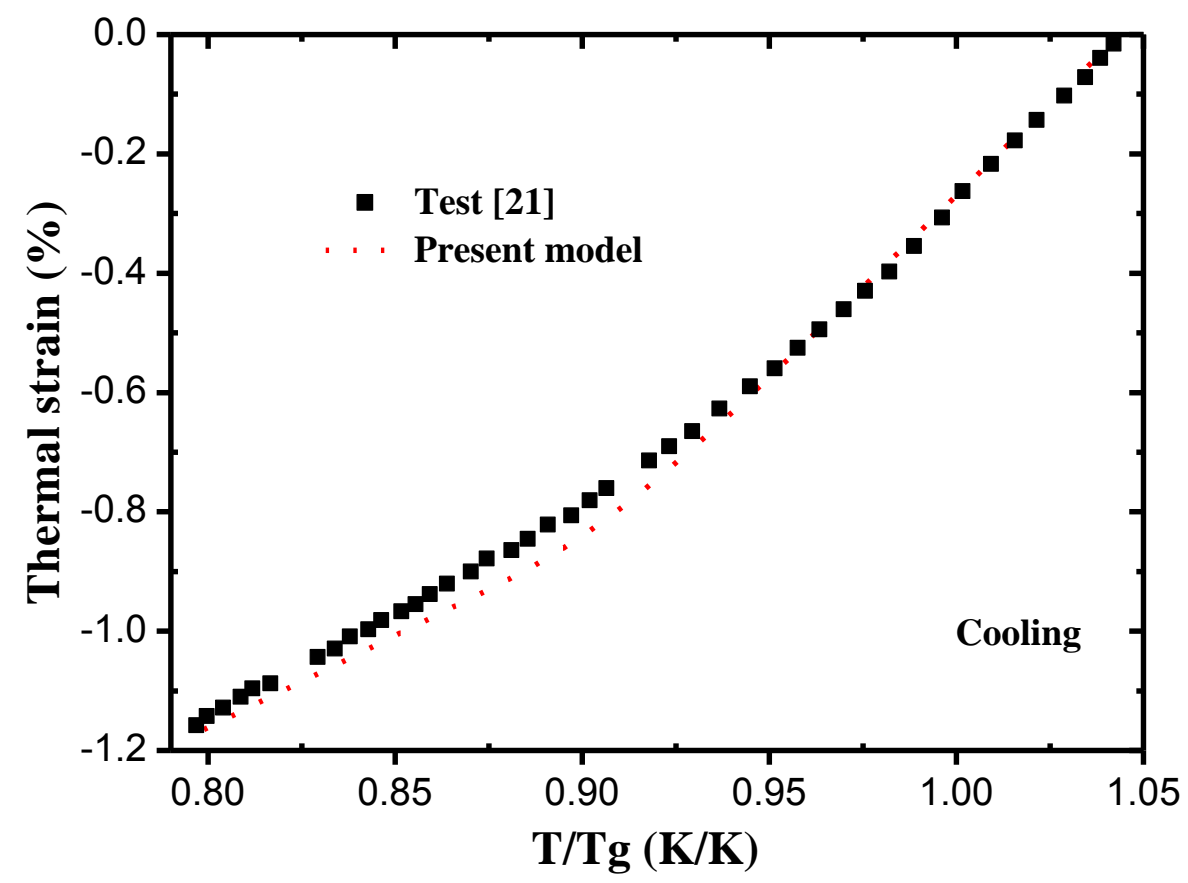

Fig 10 The thermal expansion strain-temperature curves obtained by Liu's experimentation and the calculation of the new model.

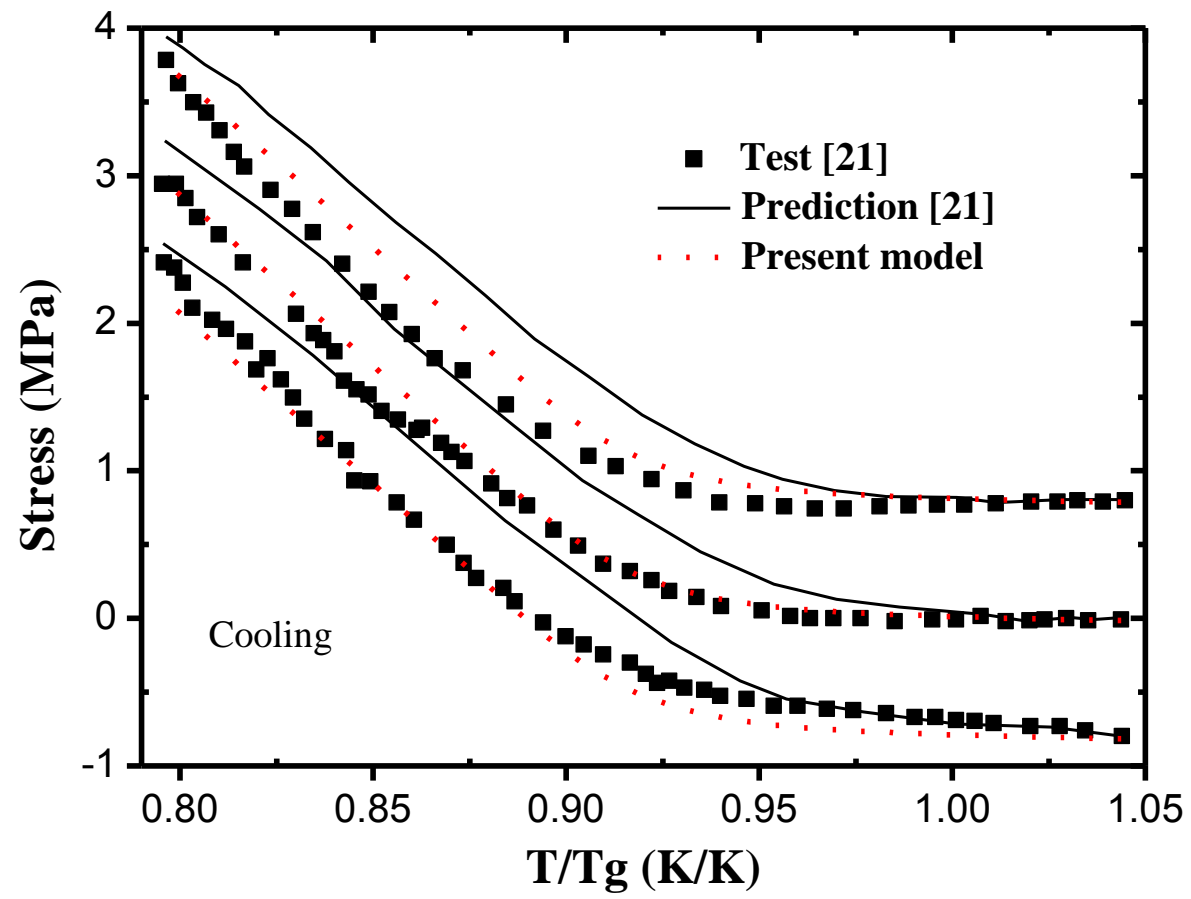

Fig 11 The stress-temperature curves obtained by Liu's experimentation, calculation and the calculation of the new model in cooling process. 


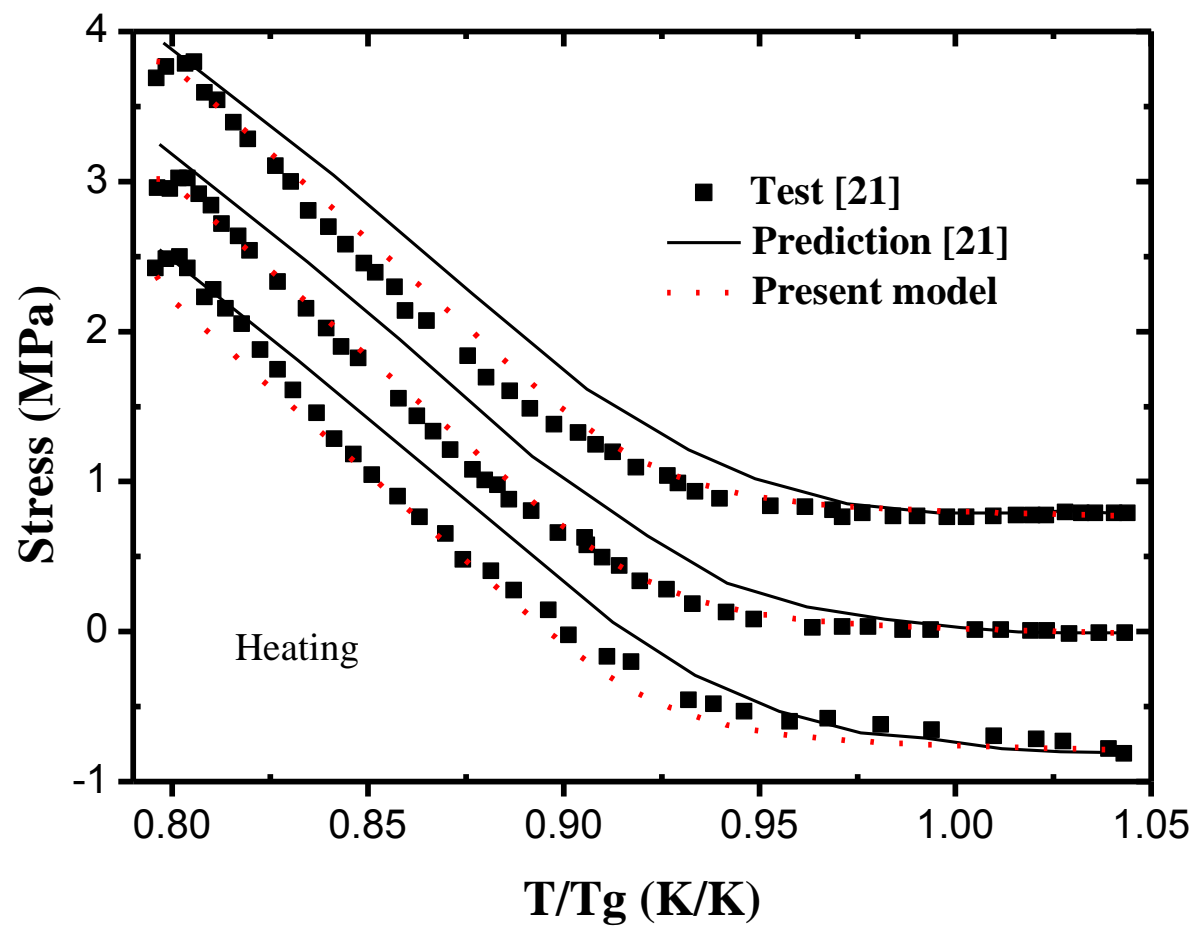

Fig 12 The stress-temperature curves obtained by Liu's experimentation, calculation and the calculation of the new model in heating process. 